Preprint

CHIBA-EP-106

hep-th/9805153

May 1998

\title{
Abelian Magnetic Monopole Dominance in Quark Confinement *
}

\author{
Kei-Ichi Kondo ${ }^{1, \dagger}$ \\ ${ }^{1}$ Department of Physics, Faculty of Science, Chiba University, Chiba 263-8522, Japan \\ † E-mail: kondo@cuphd.nd.chiba-u.ac.jp
}

\begin{abstract}
We prove Abelian magnetic monopole dominance in the string tension of QCD. Abelian and monopole dominance in low energy physics of QCD has been confirmed for various quantities by recent Monte Carlo simulations of lattice gauge theory. In order to prove this dominance, we use the reformulation of continuum Yang-Mills theory in the maximal Abelian gauge as a deformation of a topological field theory of magnetic monopoles, which was proposed in the previous article by the author. This reformulation provides an efficient way for incorporating the magnetic monopole configuration as a topological non-trivial configuration in the functional integral. We derive a version of the non-Abelian Stokes theorem and use it to estimate the expectation value of the Wilson loop. This clearly exhibits the role played by the magnetic monopole as an origin of the Berry phase in the calculation of the Wilson loop in the manifestly gauge invariant manner. We show that the string tension derived from the diagonal (abelian) Wilson loop in the topological field theory (studied in the previous article) converges to that of the full non-Abelian Wilson loop in the limit of large Wilson loop. Therefore, within the above reformulation of QCD, this result (together with the previous result) completes the proof of quark confinement in QCD based on the criterion of the area law of the full non-Abelian Wilson loop.
\end{abstract}

Key words: quark confinement, topological field theory, magnetic monopole, nonAbelian Stokes theorem

PACS: 12.38.Aw, 12.38.Lg

* To be published in Phys. Rev. D. 


\section{Introduction}

In a series of articles [1], 2, 3], we have investigated quark (resp. charge) confinement in four-dimensional non-Abelian [1, 2] (resp. Abelian [3]) gauge theories. The main purpose of them was to clarify the mechanism of quark (resp. charge) confinement and to give the proof of quark confinement starting from quantum chromodynamics (QCD) (resp. quantum electrodynamics (QED)) without introducing ad hoc assumptions. A special gauge fixing called the maximal Abelian gauge (MAG) has been adopted in these investigations. For a non-Abelian gauge group $G$, the MAG implies a partial gauge fixing in which the coset $G / H$ is fixed with the maximal torus subgroup $H$ being unbroken. The MAG is regarded as a field theoretical realization of the Abelian projection proposed by 't Hooft [4].

In the first article [1], we have proved that the QCD vacuum is the dual superconductor 1 in the sense that the low-energy effective gauge theory of QCD in the MAG is given exactly by the dual Ginzburg-Landau theory, which we called the Abelianprojected effective gauge theory (APEGT). This result supports magnetic monopole condensation as a mechanism of quark confinement. The dual superconductivity in QCD gives the most intuitively appealing picture of quark confinement.

In the second article [2], we have presented a reformulation of the non-Abelian gauge theory as a (perturbative) deformation of a topological (quantum) field theory (T(Q)FT) which describes topological non-trivial sector of the gauge theory. This reformulation provides an efficient way for incorporating the magnetic monopole [6, 7] configuration (which appears after Abelian projection) as a topological non-trivial configuration in the functional integral of gauge theory. In the article [2] we have defined the diagonal Abelian Wilson loop by using the gauge field variable belonging to the maximal torus subgroup $H$ of $G$. We have proved that, in the TFT obtained from the four-dimensional Yang-Mills (YM) theory with a gauge group $G$ in the MAG, the evaluation of the diagonal Abelian Wilson loop is reduced to that of the equivalent two-dimensional coset $G / H$ non-linear sigma model (NLSM). This equivalence is a consequence of the Parisi-Soulous dimensional reduction of the four-dimensional TFT in the MAG into the two-dimensional $G / H$ coset NLSM. This is an exact result. This result stems from the supersymmetry hidden in the TFT in the MAG. Moreover, we have shown that the area law of the diagonal Wilson loop is derived by summing up the contribution of instanton and anti-instanton configurations in the two-dimensional NLSM. These results lead to the linear confining static potential between quark and anti-quark in the TFT sector. For $G=S U(2)$, the equivalent model of the TFT is given by the $\mathrm{O}(3)$ NLSM or $C P^{1}$ model. Thus the dimensional reduction is considered as another mechanism for quark confinement.

Similar idea can also be applied to Abelian gauge theory. Actually, in the third article [3], the existence of confinement phase in the strong coupling region of QED

\footnotetext{
${ }^{1}$ According to the recent Monte Carlo simulation, the type of dual superconductor as the QCD vacuum is reported to be on the border of the type II, see [5] for the defintion of the type of dual superconductor. This will be due to the dressing of the Abelian flux connecting the quark and antiquark pair by the off-diagonal gluon components, since the Abelian dual Ginzburg-Landau theory obtained as the APEGT is of type II (near the London limit) [1].
} 
has been shown in the sense that the linear static potential is generated between two fractional charges due to vortex condensation.

As a background of the works [1, 2, 3], it is necesarry to know that the Abelian and monopole dominance [8, 9] in low energy physics in QCD has been confirmed for various quantities by recent Monte Carlo simulations of lattice gauge theory, see e.g. [10] and [11]. This is especially remarkable in the MAG. According to the lattice Monte Carlo simulations, the non-Abelian string tension $\sigma$ is nearly saturated by the Abelian part $\sigma_{\text {Abel }}$ obtained in the MAG; Indeed, $\sigma_{\text {Abel }} \cong 0.92 \sigma$ for $G=S U(2), \beta=$ 2.5115 [12]. This is called the Abelian dominance. Moreover, the Abelian part $\sigma_{\text {Abel }}$ is dominated by the monopole contribution, $\sigma_{\text {monopole }}$ as $\sigma_{\text {monopole }} \cong 0.95 \sigma_{\text {Abel }} 13$. This is called the monopole dominance. However, it is not clear whether the abelian and monopole dominance on the lattice survives the continuum limit.

In this article, to avoid the subtle problem of taking the continuum limit of the lattice gauge theory, we make use of the continuum formulation introduced in [2] of the gauge theory to study the Abelian and monopole dominance in QCD. Here it is important to remember that the criterion of quark confinement should be gauge invariant, since only the gauge invariant concept has physical meaning in gauge theories. Indeed, the full non-Abelian Wilson loop is gauge invariant by construction and hence the expectation value is independent of the gauge chosen. Therefore the area law of the full non-Abelian Wilson loop gives a gauge independent criterion for quark confinement. Consequently, the string tension obtained from the area law is gaugeinvariant and gives the gauge-independent linear static potential between quark and anti-quark. Therefore, in the practical calculation of the full non-Abelian Wilson loop, we can adopt an adequate gauge so as to simplify the calculation. It turns out that such a simplest gauge is given by the MAG.

In this article we deal with the full non-Abelian Wilson loop and clarify the relationship between the full non-Abelian Wilson loop and the diagonal Wilson loop introduced and evaluated in [2]. At first glance, it seems that the area law derived in [2] from the Abelian (diagonal) Wilson loop might depend on the specific gauge fixing chosen, the MAG. This is not the case, as shown in this article.

The main purpose of this article is to show that the area law of the diagonal Wilson loop in the TFT is sufficient to conclude the area law of the full non-Abelian Wilson loop in the YM theory. Actually, it turns out that the string tension $\sigma_{M A G}$ derived from the diagonal (abelian) Wilson loop in the TFT (studied in the previous article [2]) converges to the string tension $\sigma$ of the full non-Abelian Wilson loop in the YM theory in the limit of large Wilson loop $C$, that is to say, the difference between two string tensions goes to zero in the large Wilson loop limit,

$$
\sigma-\sigma_{M A G} \searrow 0 \text { as }|\operatorname{Area}(C)| \nearrow \infty \text {. }
$$

This impiles Abelian and monopole dominance in the string tension of QCD. Moreover, within the reformulation of gauge field theories given in [2], the result (1.1) completes the proof of quark confinement in QCD based on the criterion of the area law of full non-Abelian Wilson loop, since the area law for the diagonal Wilson loop, i.e. $\sigma_{A b e l}=\sigma_{M A G} \neq 0$ for any value of the gauge coupling $(g>0)$ was shown using dimensional reduction and instanton calculus in the previous article [2]. Under 
the MAG, we can show without ad hoc assumptions that the dual superconductivity and the dimensional reduction are exactly realized in QCD, both of which lead to monopole condensations as the mechanism for quark confinement.

This article is organized as follows. In section 2, we review the formulation of the YM theory as a deformation of the TFT [2]. In section 3, we rederive a version [18, 19] of the non-Abelian Stokes theorem (NAST) [14, 15, 16, 17, 18, 19, 20, 21] based on the coherent state representation [22, 23, 24, 25. This clearly shows gauge invariance of the Wilson loop and the role played by the magnetic monopole in the calculation of the Wilson loop. The NAST clarify also the relationship between the monopole contribution and the Berry phase [26, 27, 28, 29, 30, 31, 32]. In section 4, the NAST is used it to estimate the expectation value of the Wilson loop and to prove the main statement.

\section{Yang-Mills theory as a deformation of a TFT and dimensional reduction}

In the previous article [2], we have presented the reformulation of the non-Abelian gauge theory as a deformation of a topological field theory. In this section, we summarize the essence of this reformulation for later convenience.

\subsection{Separation of field variables}

The Yang-Mills (YM) theory with a gauge group $G=S U(N)$ on the $D$-dimensional space-time is described by the action $(D>2)$,

$$
\begin{aligned}
S_{Q C D}^{\text {tot }} & =\int d^{D} x\left(\mathcal{L}_{Q C D}\left[\mathcal{A}_{\mu}, \psi\right]+\mathcal{L}_{G F}\right), \\
\mathcal{L}_{Q C D}\left[\mathcal{A}_{\mu}, \psi\right] & :=-\frac{1}{2} \operatorname{tr}_{G}\left(\mathcal{F}_{\mu \nu} \mathcal{F}_{\mu \nu}\right)+\bar{\psi}\left(i \gamma^{\mu} \mathcal{D}_{\mu}[\mathcal{A}]-m\right) \psi,
\end{aligned}
$$

where $\mathcal{L}_{G F}$ is the gauge fixing term specified below and

$$
\begin{aligned}
\mathcal{A}_{\mu}(x) & =\sum_{A=1}^{N^{2}-1} \mathcal{A}_{\mu}^{A}(x) T^{A} \\
\mathcal{F}_{\mu \nu}(x) & :=\sum_{A=1}^{N^{2}-1} \mathcal{F}_{\mu \nu}^{A}(x) T^{A}:=\partial_{\mu} \mathcal{A}_{\nu}(x)-\partial_{\nu} \mathcal{A}_{\mu}(x)-i g\left[\mathcal{A}_{\mu}(x), \mathcal{A}_{\nu}(x)\right] \\
\mathcal{D}_{\mu}[\mathcal{A}] & :=\partial_{\mu}-i g \mathcal{A}_{\mu} .
\end{aligned}
$$

We adopt the following convention. The generators $T^{A}\left(A=1, \cdots, N^{2}-1\right)$ of the Lie algebra $\mathcal{G}$ of the gauge group $G=S U(N)$ are hermitian and satisfy $\left[T^{A}, T^{B}\right]=$ if ${ }^{A B C} T^{C}$, with a normalization, $\operatorname{tr}\left(T^{A} T^{B}\right)=\frac{1}{2} \delta^{A B}$. Let $H=U(1)^{N-1}$ be the maximal torus group of $G$ and $T^{a}$ be the generators in the Lie algebra $\mathcal{G} \backslash \mathcal{H}$ where $\mathcal{H}$ is the Lie algebra of $H$.

In the following, we discuss only the case of $S U(2)$ explicitly, although most of the following results can be easily extended into $S U(N), N>2$. For $G=S U(2), T^{A}=$ 
$(1 / 2) \sigma^{A}(A=1,2,3)$ with Pauli matrices $\sigma^{A}$ and the structure constant is $f^{A B C}=$ $\epsilon^{A B C}$. The indices $a, b, \cdots$ denote the off-diagonal parts of the matrix representation. The Cartan decomposition of the gauge field reads

$$
\mathcal{A}_{\mu}(x)=\sum_{A=1}^{3} \mathcal{A}_{\mu}^{A}(x) T^{A}:=a_{\mu}(x) T^{3}+\sum_{a=1}^{2} A_{\mu}^{a}(x) T^{a} .
$$

Under the gauge transformation, the gauge field $\mathcal{A}_{\mu}(x)$ transforms as

$$
\mathcal{A}_{\mu}(x) \rightarrow \mathcal{A}_{\mu}^{U}(x):=U(x) \mathcal{A}_{\mu}(x) U^{\dagger}(x)+\frac{i}{g} U(x) \partial_{\mu} U^{\dagger}(x) .
$$

In order to quantize the YM theory, this gauge degrees of freedom must be fixed by the procedure of gauge fixing. The gauge fixing condition is usually written as $F[\mathcal{A}]=0$. The procedure of gauge fixing must be done in such a way that the gauge fixing condition is preserved also for the gauge rotated field $\mathcal{A}_{\mu}^{U}$, i.e., $F\left[\mathcal{A}^{U}\right]=0$. This is guaranteed by the Faddeev-Popov (FP) ghost field. In the BRST formalism, both the gauge-fixing and the FP terms are automatically produced using a functional $G_{g f}$ of the field variables as

$$
\mathcal{L}_{G F}:=-i \delta_{B} G_{g f}\left[\mathcal{A}_{\mu}, \mathcal{C}, \overline{\mathcal{C}}, \phi\right],
$$

where $\mathcal{C}, \overline{\mathcal{C}}$ are ghost, anti-ghost fields and $\phi$ is the Lagrange multiplier field for the gauge fixing condition. Here $\delta_{B}$ denotes the nilpotent BRST transformation $\delta_{B}\left(\delta_{B}^{2} \equiv\right.$ $0)$,

$$
\begin{aligned}
\delta_{B} \mathcal{A}_{\mu}(x) & =\mathcal{D}_{\mu}[\mathcal{A}] \mathcal{C}(x):=\partial_{\mu} \mathcal{C}(x)-i g\left[\mathcal{A}_{\mu}(x), \mathcal{C}(x)\right], \\
\delta_{B} \mathcal{C}(x) & =i g \frac{1}{2}[\mathcal{C}(x), \mathcal{C}(x)] \\
\delta_{B} \overline{\mathcal{C}}(x) & =i \phi(x) \\
\delta_{B} \phi(x) & =0 \\
\delta_{B} \psi(x) & =i g \mathcal{C}(x) \psi(x), \quad \delta_{B} \bar{\psi}(x)=-i g \mathcal{C}(x) \bar{\psi}(x) .
\end{aligned}
$$

The partition function of QCD is given by

$$
Z_{Q C D}[J]:=\int\left[d \mathcal{A}_{\mu}\right][d \mathcal{C}][d \overline{\mathcal{C}}][d \phi][d \psi][d \bar{\psi}] \exp \left\{i S_{t o t}+i S_{J}\right\},
$$

with the source term,

$$
S_{J}:=\int d^{D} x\left(\operatorname{tr}_{\mathcal{G}}\left[J^{\mu} \mathcal{A}_{\mu}+J_{c} \mathcal{C}+J_{\bar{c}} \overline{\mathcal{C}}+J_{\phi} \phi\right]+\bar{\eta} \psi+\eta \bar{\psi}\right) .
$$

To reformulate the YM theory as a deformation of a topological field theory [2], we first regard the field $\mathcal{A}_{\mu}$ and $\psi$ as the gauge transformation of the fields $\mathcal{V}_{\mu}$ and $\Psi$,

$$
\begin{aligned}
\mathcal{A}_{\mu}(x) & :=U(x) \mathcal{V}_{\mu}(x) U^{\dagger}(x)+\Omega_{\mu}(x), \quad \Omega_{\mu}(x):=\frac{i}{g} U(x) \partial_{\mu} U^{\dagger}(x), \\
\psi(x) & :=U(x) \Psi(x),
\end{aligned}
$$


where $\mathcal{V}_{\mu}$ and $\Psi$ are identified with the field variables in the perturbative sector. Furthermore we introduce new ghost field $\gamma$, anti-ghost field $\bar{\gamma}$ and the multiplier field $\beta$ which are subject to a new BRST transformation $\tilde{\delta}_{B}$,

$$
\begin{aligned}
\tilde{\delta}_{B} \mathcal{V}_{\mu}(x) & =\mathcal{D}_{\mu}[\mathcal{V}] \gamma(x):=\partial_{\mu} \gamma(x)-i g\left[\mathcal{V}_{\mu}(x), \gamma(x)\right], \\
\tilde{\delta}_{B} \gamma(x) & =i g \frac{1}{2}[\gamma(x), \gamma(x)], \\
\tilde{\delta}_{B} \bar{\gamma}(x) & =i \beta(x), \\
\tilde{\delta}_{B} \beta(x) & =0 \\
\tilde{\delta}_{B} \Psi(x) & =i g \gamma(x) \Psi(x), \quad \tilde{\delta}_{B} \bar{\Psi}(x)=-i g \gamma(x) \bar{\Psi}(x) .
\end{aligned}
$$

Then the partition function is rewritten as

$$
\begin{aligned}
Z_{Q C D}[J]= & \int[d U][d \mathcal{C}][d \overline{\mathcal{C}}][d \phi] \int\left[d \mathcal{V}_{\mu}\right][d \gamma][d \bar{\gamma}][d \beta][d \Psi][d \bar{\Psi}] \\
& \times \exp \left\{i \int d^{D} x\left[-i \delta_{B} G_{g f}\left[\Omega_{\mu}+U \mathcal{V}_{\mu} U^{\dagger}, \mathcal{C}, \overline{\mathcal{C}}, \phi\right]\right]\right. \\
& \left.\quad+i \int d^{D} x\left[\mathcal{L}_{Q C D}\left[\mathcal{V}_{\mu}, \Psi\right]-i \tilde{\delta}_{B} \tilde{G}_{g f}\left(\mathcal{V}_{\mu}, \gamma, \bar{\gamma}, \beta\right)\right]+i S_{J}\right\}
\end{aligned}
$$

where

$$
S_{J}=\int d^{D} x\left\{\operatorname{tr}_{\mathcal{G}}\left[J^{\mu}\left(\Omega_{\mu}+U \mathcal{V}_{\mu} U^{\dagger}\right)+J_{c} \mathcal{C}+J_{\bar{c}} \overline{\mathcal{C}}+J_{\phi} \phi\right]+\bar{\eta} U \Psi+\eta \bar{\Psi} U^{\dagger}\right\} .
$$

\subsection{Maximal Abelian gauge}

A covariant choice for gauge fixing is the Lorentz gauge,

$$
F[\mathcal{A}]:=\partial_{\mu} \mathcal{A}^{\mu}=0 .
$$

The most familiar choice of $G_{g f}$ is

$$
G_{g f}=\operatorname{tr}_{\mathcal{G}}\left[\overline{\mathcal{C}}\left(\partial_{\mu} \mathcal{A}^{\mu}+\frac{\alpha}{2} \phi\right)\right]
$$

which yields

$$
\mathcal{L}_{G F}:=-i \delta_{B} G_{g f}\left[\mathcal{A}_{\mu}, \mathcal{C}, \overline{\mathcal{C}}, \phi\right]=\operatorname{tr}_{\mathcal{G}}\left[\phi \partial_{\mu} \mathcal{A}^{\mu}+i \overline{\mathcal{C}} \partial^{\mu} \mathcal{D}_{\mu}[\mathcal{A}] \mathcal{C}+\frac{\alpha}{2} \phi^{2}\right] .
$$

The parameter $\alpha$ is called the gauge-fixing parameter.

In the previous articles [1, 2], we examined the maximal abelian gauge (MAG). For $G=S U(2)$, MAG is given by

$$
F^{ \pm}[A, a]:=\left(\partial^{\mu} \pm i g a^{\mu}\right) A_{\mu}^{ \pm}=0
$$

using the $( \pm, 3)$ basis,

$$
\mathcal{O}^{ \pm}:=\left(\mathcal{O}^{1} \pm i \mathcal{O}^{2}\right) / \sqrt{2}
$$


The simplest choice of $G_{g f}$ for MAG in $( \pm, 3)$ basis is

$$
G_{g f}=\sum_{ \pm} \bar{C}^{\mp}\left(F^{ \pm}[A, a]+\frac{\alpha}{2} \phi^{ \pm}\right)
$$

which is equivalently rewritten in the usual basis as

$$
\begin{aligned}
G_{g f} & =\sum_{a=1,2} \bar{C}^{a}\left(F^{a}[A, a]+\frac{\alpha}{2} \phi^{a}\right), \\
F^{a}[A, a] & :=\left(\partial^{\mu} \delta^{a b}-g \epsilon^{a b 3} a^{\mu}\right) A_{\mu}^{b}:=D^{\mu a b}[a] A_{\mu}^{b} .
\end{aligned}
$$

In the previous article [2], we took a slightly modified choice,

$$
G_{g f}^{\prime}=-\bar{\delta}_{B}\left(\frac{1}{2} A_{\mu}^{a} A^{\mu a}+i C^{a} \bar{C}^{a}\right)=-\bar{\delta}_{B}\left(A_{\mu}^{+} A_{\mu}^{-}+i \sum_{ \pm} C^{ \pm} \bar{C}^{\mp}\right),
$$

where $\bar{\delta}_{B}$ is the anti-BRST transformation,

$$
\begin{aligned}
\bar{\delta}_{B} \mathcal{A}_{\mu}(x) & =\mathcal{D}_{\mu}[\mathcal{A}] \overline{\mathcal{C}}(x):=\partial_{\mu} \overline{\mathcal{C}}(x)-i g\left[\mathcal{A}_{\mu}(x), \overline{\mathcal{C}}(x)\right], \\
\bar{\delta}_{B} \mathcal{C}(x) & =i \bar{\phi}(x), \\
\bar{\delta}_{B} \overline{\mathcal{C}}(x) & =i g \frac{1}{2}[\overline{\mathcal{C}}(x), \overline{\mathcal{C}}(x)], \\
\bar{\delta}_{B} \bar{\phi}(x) & =0, \\
\bar{\delta}_{B} \psi(x) & =i g \overline{\mathcal{C}}(x) \psi(x), \quad \bar{\delta}_{B} \bar{\psi}(x)=-i g \overline{\mathcal{C}}(x) \bar{\psi}(x), \\
\phi(x)+\bar{\phi}(x) & =g[\mathcal{C}(x), \overline{\mathcal{C}}(x)],
\end{aligned}
$$

where $\bar{\phi}$ is defined in the last equation. The BRST and anti-BRST transformations have the following properties,

$$
\left(\delta_{B}\right)^{2}=0, \quad\left(\bar{\delta}_{B}\right)^{2}=0, \quad\left\{\delta_{B}, \bar{\delta}_{B}\right\}:=\delta_{B} \bar{\delta}_{B}+\bar{\delta}_{B} \delta_{B}=0
$$

Our choice of gauge fixing term leads to a remarkable form for the gauge-fixing part,

$$
\mathcal{L}_{G F}=i \delta_{B} \bar{\delta}_{B}\left(\frac{1}{2} A_{\mu}^{a} A^{\mu a}+i C^{a} \bar{C}^{a}\right)=i \delta_{B} \bar{\delta}_{B}\left(A_{\mu}^{+} A_{\mu}^{-}+i \sum_{ \pm} C^{ \pm} \bar{C}^{\mp}\right)
$$

which is invariant under the BRST and anti-BRST transformations,

$$
\delta_{B} \mathcal{L}_{G F}=0=\bar{\delta}_{B} \mathcal{L}_{G F}
$$

The choice of $G_{g f}^{\prime}$ allows the separation of the variable in such a way

$$
\begin{aligned}
\mathcal{L}_{G F}= & -i \delta_{B} G_{g f}^{\prime}\left[\Omega_{\mu}+U \mathcal{V}_{\mu} U^{\dagger}, \mathcal{C}, \overline{\mathcal{C}}, \phi\right] \\
& =\mathcal{L}_{T F T}\left[\Omega_{\mu}, \mathcal{C}, \overline{\mathcal{C}}, \phi\right]+i \mathcal{V}_{\mu}^{A} \mathcal{M}_{\mu}^{A}[U]+\frac{i}{2} \mathcal{V}_{\mu}^{A} \mathcal{V}_{\mu}^{B} \mathcal{K}^{A B}[U]
\end{aligned}
$$


where we have defined,

$$
\begin{aligned}
\mathcal{L}_{T F T} & :=-i \delta_{B} G_{g f}^{\prime}\left[\Omega_{\mu}, \mathcal{C}, \overline{\mathcal{C}}, \phi\right]=i \delta_{B} \bar{\delta}_{B}\left(\frac{1}{2} \Omega_{\mu}^{a} \Omega_{\mu}^{a}+i C^{a} \bar{C}^{a}\right) \\
\mathcal{M}_{\mu}^{A}[U] & :=\delta_{B} \bar{\delta}_{B}\left[\left(U T^{A} U^{\dagger}\right)^{a} \Omega_{\mu}^{a}\right] \\
\mathcal{K}^{A B}[U] & :=\delta_{B} \bar{\delta}_{B}\left[\left(U T^{A} U^{\dagger}\right)^{a}\left(U T^{B} U^{\dagger}\right)^{a}\right]
\end{aligned}
$$

where we have used that the action of $\delta_{B}$ is trivial in the perturbative sector,

$$
\delta_{B} \mathcal{V}_{\mu}(x)=0=\bar{\delta}_{B} \mathcal{V}_{\mu}(x)
$$

The most basic BRST transformation $\delta_{B}$ is given by

$$
\delta_{B} U(x)=i g \mathcal{C}(x) U(x), \quad \bar{\delta}_{B} U(x)=i g \overline{\mathcal{C}}(x) U(x)
$$

which yields

$$
\delta_{B} \Omega_{\mu}(x)=\mathcal{D}_{\mu}[\Omega] \mathcal{C}(x), \quad \bar{\delta}_{B} \Omega_{\mu}(x)=\mathcal{D}_{\mu}[\Omega] \overline{\mathcal{C}}(x)
$$

\subsection{Deformation of topological field theory}

The partition function of QCD is rewritten as

$$
\begin{aligned}
& Z_{Q C D}[J]:=\int[d U][d \mathcal{C}][d \overline{\mathcal{C}}][d \phi] \exp \left\{i S_{T F T}\left[\Omega_{\mu}, \mathcal{C}, \overline{\mathcal{C}}, \phi\right]\right. \\
& \left.+i \int d^{D} x \operatorname{tr}_{\mathcal{G}}\left[J^{\mu} \Omega_{\mu}+J_{c} \mathcal{C}+J_{\bar{c}} \overline{\mathcal{C}}+J_{\phi} \phi\right]+i W\left[U ; J^{\mu}, \bar{\eta}, \eta\right]\right\}, \\
& e^{i W\left[U ; J^{\mu}, \bar{\eta}, \eta\right]}:=\int\left[d \mathcal{V}_{\mu}\right][d \gamma][d \bar{\gamma}][d \beta][d \Psi][d \bar{\Psi}] \exp \left\{i S_{p Q C D}\left[\mathcal{V}_{\mu}, \Psi, \gamma, \bar{\gamma}, \beta\right]\right. \\
& +i \int d^{D} x\left[\mathcal{V}_{\mu}^{A} \mathcal{J}_{\mu}^{A}+\frac{i}{2} \mathcal{V}_{\mu}^{A} \mathcal{V}_{\mu}^{B} \mathcal{K}^{A B}[U]\right. \\
& \left.\left.+\operatorname{tr}_{\mathcal{G}}\left(\bar{\eta} U \Psi+\eta \bar{\Psi} U^{\dagger}\right)\right]\right\} \\
& S_{p Q C D}\left[\mathcal{V}_{\mu}, \Psi, \gamma, \bar{\gamma}, \beta\right]:=\int d^{D} x\left[\mathcal{L}_{Q C D}[\mathcal{V}, \Psi]-i \tilde{\delta}_{B} \tilde{G}_{g f}\left(\mathcal{V}_{\mu}, \gamma, \bar{\gamma}, \beta\right)\right] \text {, } \\
& \mathcal{J}_{\mu}^{A}:=\left(U^{\dagger} J^{\mu} U\right)^{A}+i \mathcal{M}_{\mu}^{A}[U],
\end{aligned}
$$

where $[d U]$ is the invariant measure on the group $G$. Here $W\left[U ; J^{\mu}, \bar{\eta}, \eta\right]$ denotes the deformation from the TFT. When $U \equiv 1$ and $\mathcal{M}_{\mu}^{A}[U] \equiv 0 \equiv \mathcal{K}^{A B}[U], W\left[U ; J^{\mu}, \bar{\eta}, \eta\right]$ coincides with the generating functional of the connected correlation function in the perturbative QCD (pQCD) with the action $S_{p Q C D}$ (topological trivial sector). The correlation functions of the original fundamental field $\mathcal{A}_{\mu}, \psi, \bar{\psi}$ is obtained by differentiating $Z_{Q C D}[J]$ with respect to the source $J_{\mu}, \bar{\eta}, \eta$. The sector written in terms of the TFT fields $(U, \mathcal{C}, \overline{\mathcal{C}}, \phi)$ should be treated non-perturbatively. The perturbative expansion around the TFT means the integration over the new fields $\left(\mathcal{V}_{\mu}, \gamma, \bar{\gamma}, \beta\right)$ based on the perturbative expansion in powers of the coupling constant $g$. The deformation $W\left[U ; J^{\mu}, \bar{\eta}, \eta\right]$ should be calculated according to the ordinary perturbation theory in the coupling constant $g$, keeping the variable $U$ untouched. An interpretation of this reformulation was given from the viewpoint of the background field method [33]. 


\subsection{Dimensional reduction to NLSM}

For a while, we neglect the perturbative contribution $W$ and consider only the TFT part. Owing to the gauge choice of MAG, Parisi-Soulas dimensional reduction occurs for the TFT. Consequently, the D-dimensional TFT with the action

$$
S_{T F T}\left[\Omega_{\mu}, \mathcal{C}, \overline{\mathcal{C}}, \phi\right]=\int d^{D} x i \delta_{B} \bar{\delta}_{B}\left(\frac{1}{2} \Omega_{\mu}^{a}(x) \Omega_{\mu}^{a}(x)+i C^{a}(x) \bar{C}^{a}(x)\right)
$$

is equivalent to the (D-2)-dimensional coset $\mathrm{G} / \mathrm{H}$ non-linear sigma model (NLSM) with an action,

$$
\begin{aligned}
S_{N L S M}[U] & =2 \pi \int d^{D-2} z \frac{1}{2} \Omega_{\mu}^{a}(z) \Omega_{\mu}^{a}(z), \quad \Omega_{\mu}(z):=\frac{i}{g} U(z) \partial_{\mu} U^{\dagger}(z) \\
& =\frac{\beta}{2} \int d^{D-2} z \operatorname{tr}_{\mathcal{G} \backslash \mathcal{H}}\left[\partial^{\mu} U(z) \partial^{\mu} U^{\dagger}(z)\right], \quad \beta:=\frac{2 \pi}{g^{2}} .
\end{aligned}
$$

Therefore, the TFT part of four-dimensional SU(2) non-Abelian gauge theory is reduced to the two-dimensional O(3) NLSM. Hence, the calculation of the diagonal Wilson loop for the four-dimensional topological part is reduced to that in the twodimensional $\mathrm{O}(3)$ NLSM or equivalent $C P^{1}$ model.

In the previous article [2], the area law decay of the expectation value of the Wilson loop in the four-dimensional TFT (as a topological non-trivial sector of the four-dimensional YM theory) has been deduced by summing up the instanton and anti-instanton configurations in the two-dimensional equivalent NLSM and the linear confining static potential between quark and anti-quark in the TFT sector is derived. In this article, we show that the area law of the diagonal Wilson loop in the TFT is sufficient to conclude the area law of the full non-Abelian Wilson loop in the YM theory. This completes the proof of quark confinement based on the criterion of the Wilson loop in the four-dimensional YM theory.

\section{Non-Abelian Stokes theorem}

The Wilson loop operator is defined as a path ordered product of an exponent along a closed loop $C$. In the Abelian case, due to the ordinary Stokes theorem, it is rewritten as a surface integral on the surface $S$ whose boundary is given by $C$. In contrast to the ordinary Stokes theorem, there may be many possibilities for the nonAbelian Stokes theorem (NAST) [14, 15, 16, 17, 18, 19, 20, 21]. In this article we treat a version of the NAST derived by Diakonov and Petrov [18, 19. This version of NAST is able to remove path ordering from the expression of the non-Abelian Wilson loop. Instead, we must perform the functional integration. First of all, we rederive the NAST for the gauge group $G=S U(2)$ using the path integral formalism in the spin coherent state representation. Moreover, we clarify the relationship between the induced magnetic monopole and the Berry phase which appear in the NAST. Second, we give the general NAST for any compact Lie group $G$. The NAST is manifestly gauge invariant as in the Wilson loop. In the next section, we make use it to prove the abelian monopole dominance in the string tension of QCD. 


\subsection{Path integral in the coherent state representation}

We consider the formal expression,

$$
Z[t, 0]:=\operatorname{tr} \mathcal{P}_{t} \exp \left[-i \int_{0}^{t} d \tau \mathcal{H}(\tau)\right]
$$

where $\mathcal{P}_{\tau}$ is the $t$-ordering (or path-ordering) operator and the "Hamiltonian" $\mathcal{H}$ is specified later. We can make it well defined by taking the limit of discretization,

$$
Z[t, 0]=\lim _{N \rightarrow \infty, \epsilon \rightarrow 0} \operatorname{tr} \mathcal{P}_{\tau} \prod_{n=0}^{N-1}\left[1-i \epsilon \mathcal{H}\left(\tau_{n}\right)\right],
$$

where $\epsilon=t / N$ is the timestep and $\tau_{n}=n \epsilon$ is the discrete time. The limit is taken keeping $N \epsilon=t$ constant. For a given Hamiltonian in the representation $J$, we would like to obtain a path integral representation of the partition function for a spin system.

We make use of the spin coherent state to write the path integral representation of $Z[t, 0]$. Consider the group $\mathrm{SU}(2)$ and an irreducible representation characterized by highest spin $J$. Let $|0\rangle$ denote the maximally polarized state $|0\rangle=|J, J\rangle$ which is the highest weight state of a spin- $J$ representation $\{|J, M\rangle\}$ of $S U(2)$ where $M$ labels the eigenvalue of $J_{3}$,

$$
\begin{aligned}
\mathbf{J}^{2}|J, M\rangle & =J(J+1)|J, M\rangle, \quad(-J \leq M \leq J), \\
J_{3}|J, M\rangle & =M|J, M\rangle .
\end{aligned}
$$

The state $|J, M\rangle$ is an eigenvector of both the diagonal generator $J_{3}$ (Cartan subalgebra) and the quadratic Casimir invariant $\mathbf{J}^{2}$. Spin coherent sates are a family of spin state $\{|\mathbf{n}\rangle\}$ which is obtained by applying the rotation operator $R$ to the maximally polarized state $|0\rangle$,

$$
|\mathbf{n}\rangle:=R(\chi, \theta, \varphi)|J, J\rangle=e^{i J^{3} \varphi} e^{i J^{2} \theta} e^{i J^{3} \chi}|J, J\rangle,
$$

where $J^{A}(A=1,2,3)$ are three generators of $\mathrm{SU}(2)$ and $(\chi, \varphi, \theta)$ are Euler angles and the unit vector $\mathbf{n}$ parameterizes the spin coherent state. We have the freedom to define $\chi$ arbitrary. This is a $\mathrm{U}(1)$ gauge freedom. We can eliminate it by fixing $\chi$. This is the gauge fixing for the residual gauge group $H=U(1)$. The states are in oneto-one correspondence with the (right) coset $S U(2) / U(1)$ where $U(1)$ is generated by $J_{3}$ (rotation about the $z$ axis). In the language of differential geometry, the coherent states form a Hermitian line bundle associated with the Hopf, or monopole, principal bundle.

The diagonal matrix element of the generators reads

$$
\left\langle\mathbf{n}\left|J^{A}\right| \mathbf{n}\right\rangle=J n^{A}
$$

where

$$
\mathbf{n}(x)=\left(n^{1}(x), n^{2}(x), n^{3}(x)\right)=(\sin \theta(x) \cos \varphi(x), \sin \theta(x) \sin \varphi(x), \cos \theta(x)) .
$$


It is known [22, 25] that the coherent sates are not orthogonal. The overlap, i.e. the inner product of any two coherent states is evaluated as

$$
\begin{aligned}
\left\langle\mathbf{n} \mid \mathbf{n}^{\prime}\right\rangle & =\left(\frac{1+\mathbf{n} \cdot \mathbf{n}^{\prime}}{2}\right)^{J} e^{-i J \Phi\left(\mathbf{n}, \mathbf{n}^{\prime}\right)} \\
\Phi\left(\mathbf{n}, \mathbf{n}^{\prime}\right) & :=2 \arctan \left\{\frac{\cos \left[\frac{1}{2}\left(\theta+\theta^{\prime}\right)\right]}{\cos \left[\frac{1}{2}\left(\theta-\theta^{\prime}\right)\right]} \tan \left(\frac{\varphi-\varphi^{\prime}}{2}\right)\right\}+\chi-\chi^{\prime},
\end{aligned}
$$

where $\chi, \chi^{\prime}$ depend on the gauge fixing.

The coherent states span the space of states of spin $J$. The measure of integration over the group parameters is defined by

$$
d \mu(\mathbf{n}):=\frac{2 J+1}{4 \pi} \delta(\mathbf{n} \cdot \mathbf{n}-1) d^{3} \mathbf{n}=\frac{2 J+1}{4 \pi} \sin \theta d \theta d \varphi .
$$

This is a Haar measure of the coset $S U(2) / U(1)$, in other words, it is the area element on the two-sphere $S^{2}$. The state $|\mathbf{n}\rangle$ can be expanded in a complete basis of the spin- $J$ irreducible representation $\{|J, M\rangle\}$. The coefficients of the expansion are the representation matrix,

$$
|\mathbf{n}\rangle=\sum_{M=-J}^{+J}|J, M\rangle D_{M J}^{(J)}(\mathbf{n}) .
$$

The resolution of unity is given by

$$
\int d \mu(\mathbf{n})|\mathbf{n}\rangle\left\langle\mathbf{n}\left|=\sum_{M=-J}^{+J}\right| J, M\right\rangle\langle J, M|=I,
$$

where $I$ is an identity operator. Hence the coherent state $|\mathbf{n}\rangle$ forms the complete set, although it is not orthogonal. Thus the coherent states form an overcomplete basis.

In particular, for $J=\frac{1}{2}$, an element $U(x) \in S U(2)$ is written as follows by introducing three local field variables $(\theta(x), \varphi(x), \chi(x))$ corresponding to the Euler angles,

$$
\begin{aligned}
R(\varphi, \theta, \chi)= & U(x)=e^{i \varphi(x) \sigma_{3} / 2} e^{i \theta(x) \sigma_{2} / 2} e^{i \chi(x) \sigma_{3} / 2} \\
= & \left(\begin{array}{ll}
e^{\frac{i}{2}(\varphi(x)+\chi(x))} \cos \frac{\theta(x)}{2} & -e^{\frac{i}{2}(\varphi(x)-\chi(x))} \sin \frac{\theta(x)}{2} \\
e^{-\frac{i}{2}(\varphi(x)-\chi(x))} \sin \frac{\theta(x)}{2} & e^{-\frac{i}{2}(\varphi(x)+\chi(x))} \cos \frac{\theta(x)}{2}
\end{array}\right), \\
& \theta \in[0, \pi], \varphi \in[0,2 \pi], \chi \in[0,2 \pi],
\end{aligned}
$$

and (3.4) reads

$$
|\mathbf{n}\rangle=R(\chi, \theta, \varphi)\left(\begin{array}{l}
1 \\
0
\end{array}\right)=e^{\frac{i}{2} \chi(x)}\left(\begin{array}{c}
e^{\frac{i}{2} \varphi(x)} \cos \frac{\theta(x)}{2} \\
e^{-\frac{i}{2} \varphi(x)} \sin \frac{\theta(x)}{2}
\end{array}\right) .
$$

By making use of the explicit representation, we can make sure that the formulae (3.5), (3.8) and (3.11) hold for $J=1 / 2$. 
Inserting $N$ resolutions of unit (3.11) between the factors in (3.2), we obtain

$$
Z[t, 0]=\lim _{N \rightarrow \infty, \epsilon \rightarrow 0} \prod_{n=1}^{N} d \mu\left(\mathbf{n}\left(\tau_{n}\right)\right) \prod_{\tau=\epsilon}^{t}\langle\mathbf{n}(\tau) \mid \mathbf{n}(\tau-\epsilon)\rangle[1-i \epsilon H(\tau)],
$$

where the "classical" Hamiltonian is defined by

$$
H(\tau):=\frac{\langle\mathbf{n}(\tau)|\mathcal{H}(\tau)| \mathbf{n}(\tau-\epsilon)\rangle}{\langle\mathbf{n}(\tau) \mid \mathbf{n}(\tau-\epsilon)\rangle},
$$

and the periodic boundary condition is adopted,

$$
\mathbf{n}(t)=\mathbf{n}(0) .
$$

In the limit $N \rightarrow \infty$, we replace the differences by the corresponding derivatives,

$$
\mathbf{n}(\tau+\epsilon)-\mathbf{n}(\tau) \rightarrow \epsilon \dot{\mathbf{n}}(\tau)+O\left(\epsilon^{2}\right) .
$$

For more rigorous treatment, see [22, 23].

Using (3.8), the overlap between coherent states at nearby steps to leading order in $\epsilon$ reads

$$
\begin{aligned}
& \prod_{\tau=\epsilon}^{t}\langle\mathbf{n}(\tau+\epsilon) \mid \mathbf{n}(\tau)\rangle \\
= & \exp \left\{-i J \sum_{\tau=\epsilon}^{t} \Phi(\mathbf{n}(\tau+\epsilon), \mathbf{n}(\tau))+J \sum_{\tau=\epsilon}^{t} \ln \left[\frac{1+\mathbf{n}(\tau) \cdot \mathbf{n}(\tau-\epsilon)}{2}\right]\right\} .
\end{aligned}
$$

Making use of (3.8), we obtain

$$
\Phi(\mathbf{n}(\tau+\epsilon), \mathbf{n}(\tau)) \cong \epsilon[\dot{\varphi}(\tau) \cos \theta(\tau)+\dot{\chi}(\tau)]
$$

whereas 3.17) leads to

$$
\ln \left[\frac{1+\mathbf{n}(\tau) \cdot \mathbf{n}(\tau-\epsilon)}{2}\right] \cong \ln \left[1-\frac{\epsilon^{2}}{4}\left(\partial_{\tau} \mathbf{n}\right)^{2}\right] \cong-\frac{\epsilon^{2}}{4}\left(\partial_{\tau} \mathbf{n}\right)^{2} .
$$

Within the same approximation, the classical Hamiltonian can be evaluated at equal times,

$$
H(\tau) \rightarrow\langle\mathbf{n}(\tau)|\mathcal{H}(\tau)| \mathbf{n}(\tau)\rangle+O(\epsilon)
$$

By exponentiating the Hamiltonian and discarding higher-order terms in $\epsilon$, the formal continuum limit of (3.14) is obtained,

$$
\begin{aligned}
Z[t, 0] & =\int\left[d \mu_{C}(\mathbf{n})\right] \exp (i S[\mathbf{n}]), \\
S[\mathbf{n}] & :=-\int_{0}^{t} d \tau H[\mathbf{n}]-\gamma(t)+\frac{J}{4} \epsilon \int_{0}^{t} d \tau\left(\partial_{\tau} \mathbf{n}\right)^{2}, \\
H[\mathbf{n}] & :=\langle\mathbf{n}(\tau)|\mathcal{H}(\tau)| \mathbf{n}(\tau)\rangle, \\
\gamma(t) & :=J \int_{0}^{t} d \tau[\dot{\varphi}(\tau) \cos \theta(\tau)+\dot{\chi}(\tau)],
\end{aligned}
$$


where

$$
\left[d \mu_{C}(\mathbf{n})\right]:=\lim _{N \rightarrow \infty, \epsilon \rightarrow 0} \prod_{n=1}^{N} d \mu\left(\mathbf{n}\left(\tau_{n}\right)\right) .
$$

The first term, the Hamiltonian, is specified below. Though the last term in $S[\mathbf{n}]$ vanishes in the continuum limit $\epsilon \rightarrow 0$, it plays the role of a regularization. Without it, the 'action' $S[\mathbf{n}]$ has no 'kinetic term' for the field $\mathbf{n}$.

The second term $\gamma(t)$ depends on the trajectory of $\mathbf{n}(\tau)$ on the sphere and not on its explicit time dependence. It is geometric. The phase $\gamma(t)$ is called the Berry phase or geometric phase of the spin history [26, 27]. The Berry phase measures the area enclosed by the path $\mathbf{n}(\tau)$ on the unit sphere. The area increment is a spherical triangle with vertices at $\mathbf{n}(\tau), \mathbf{n}(\tau+\epsilon)$ and $(0,0,1)$ whose area is given by

$$
\omega:=[1-\cos \theta(\tau)] d \varphi(\tau) .
$$

Hence the total area enclosed by the closed orbit is equal to

$$
\oint_{\Gamma} \omega:=\int_{0}^{t} d \tau[1-\cos \theta(\tau)] \dot{\varphi}(\tau)
$$

The Berry phase

$$
\gamma(t)=J \Omega=J \oint_{\Gamma} \omega=4 \pi J Q
$$

is expressed in a gauge invariant form.

We can introduce a vector potential

$$
\Omega:=\int_{0}^{t} d \tau \mathbf{A}(\tau) \cdot \frac{d}{d \tau} \mathbf{n}(\tau)
$$

producing the a unit magnetic monopole whose line integral over the orbit $\mathbf{n}(\tau)$ is equal to the solid angle $\Omega$ subtended by that orbit. For example, in the domain

$$
\begin{aligned}
U_{S} & :=S^{2}-\text { South Pole }=\left\{(\theta, \varphi) \in S^{2} ; \theta \neq \pi\right\}, \\
U_{N} & :=S^{2}-\text { North Pole }=\left\{(\theta, \varphi) \in S^{2} ; \theta \neq 0\right\}, \\
U_{M} & :=S^{2}-\text { Meridian }=\left\{(\theta, \varphi) \in S^{2} ; \theta \neq 0, \pi, \varphi \neq 0\right\},
\end{aligned}
$$

the vector potential is respectively given by

$$
\begin{aligned}
\mathbf{A}_{S} & :=-\frac{1-\cos \theta}{r \sin \theta} \hat{\varphi}=-\left(-\frac{y}{r(r+z)}, \frac{x}{r(r+z)}, 0\right) \\
\mathbf{A}_{N} & :=\frac{1+\cos \theta}{r \sin \theta} \hat{\varphi}=\left(-\frac{y}{r(r-z)}, \frac{x}{r(r-z)}, 0\right) \\
\mathbf{A}_{M} & :=\frac{\cos \theta}{r \sin \theta} \hat{\varphi}=\left(-\frac{y z}{r\left(r^{2}-z^{2}\right)}, \frac{x z}{r\left(r^{2}-z^{2}\right)}, 0\right)
\end{aligned}
$$


where $\hat{\varphi}$ is a unit vector in the direction of $\varphi$. The corresponding connection one-form $\omega$ is given (for a choice of $\chi$ ) by

$$
\begin{aligned}
\omega_{S} & :=J(-\cos \theta+1) d \varphi=2 J \sin ^{2} \frac{\theta}{2} d \varphi, \quad(\chi=-\varphi), \\
\omega_{N} & :=J(-\cos \theta-1) d \varphi=-2 J \cos ^{2} \frac{\theta}{2} d \varphi, \quad(\chi=+\varphi), \\
\omega_{M} & :=-J \cos \theta d \varphi . \quad(\chi=0) .
\end{aligned}
$$

Note that $\omega_{N}$ and $\omega_{S}$ are interrelated by the gauge transformation,

$$
\omega_{S}=\omega_{N}+2 J d \varphi
$$

The connection one-form is related to the curvature two-form by the ordinary Stokes theorem,

$$
\oint_{\Gamma} \omega=\int_{S} d \omega, \quad \Gamma=\partial S
$$

The curvature two-form $d \omega$ does not depend on the choice of the connection one-form (3.35), since

$$
d \omega=J \sin \theta d \theta \wedge d \varphi
$$

The Berry phase measures the flux of magnetic monopole through the area $S$ of $S^{2}$ bounded by the trajectory $\Gamma$ of $\mathbf{n}(t)$.

Perform the contour integral along the $\theta=$ constant line for (3.36),

$$
\oint_{C} \omega_{S}=\oint_{C} \omega_{N}+2 J \oint_{C} d \varphi=\oint_{C} \omega_{N}+4 \pi J
$$

This implies

$$
e^{i \oint_{C} \omega_{S}}=e^{i \oint_{C} \omega_{N}} e^{i 4 \pi J}
$$

and $e^{i 4 \pi J}=1$, i.e., $4 \pi J=2 \pi n$. Thus the quantization of the spin $J=\frac{n}{2}$ is obtained as a topological invariant. Incidentally, the connection one-form $\omega_{S}, \omega_{N}$ is written using the unit vector as

$$
\omega(x)=J \frac{n^{1}(x) d n^{2}(x)-n^{2}(x) d n^{1}(x)}{1 \pm n_{3}(x)} .
$$

\subsection{Non-Abelian Stokes theorem for $G=S U(2)$}

Now we apply the above result to evaluate the Wilson loop operator. We consider the Hamiltonian

$$
\mathcal{H}(t)=\mathcal{A}(t):=\mathcal{A}_{\mu}(x) \frac{d x^{\mu}}{d t}=\mathcal{A}^{A}(t) T^{A}=\mathcal{A}_{\mu}^{A}(x) T^{A} \frac{d x^{\mu}}{d t},
$$


where $\mathcal{A}(t)$ is the tangent component of the YM field along the loop (see the next subsection for a more precise definition). Using (3.5), we obtain

$$
H[\mathbf{n}]=i J \mathcal{A}^{A}(t) n^{A}(t)=J \operatorname{tr}\left[\sigma_{3} U \mathcal{A} U^{\dagger}\right]
$$

and

$$
\int_{0}^{t} d \tau H[\mathbf{n}]=J \int_{0}^{t} d \tau \operatorname{tr}\left[\sigma_{3} U \mathcal{A} U^{\dagger}\right]
$$

where $\sigma_{3}$ is the third Pauli matrix and we have used the adjoint orbit representation for $\mathbf{n}$,

$$
n^{A}(x) T^{A}=U^{\dagger}(x) T^{3} U(x)
$$

Using (3.12), we can see that the unit vector $\mathbf{n}(x)$ defined by (3.45) is equal to (3.6).

On the other hand, using (3.12) the Berry phase is rewritten as

$$
\gamma(t)=J \int_{0}^{t} d \tau \operatorname{tr}\left(\sigma_{3} i U \frac{d}{d t} U^{\dagger}\right)
$$

where the functional $\gamma(t)$ denotes the phase acquired by a spin that aligns with an

adiabatically rotating external field $\overrightarrow{\mathcal{A}}$ which is parallel to $\mathbf{n}(\tau)$. Finally we have shown

$$
\begin{aligned}
Z[t, 0] & =\operatorname{tr}_{C} \exp \left[-i \oint_{C} d x^{\mu} \mathcal{A}_{\mu}(x)\right]=\int[d \mu(\mathbf{n})] \exp (i S[\mathbf{n}]), \\
S[\mathbf{n}] & :=J \int_{0}^{t} d \tau \operatorname{tr}\left\{\sigma_{3}\left(U \mathcal{A} U^{\dagger}+i U \frac{d}{d \tau} U^{\dagger}\right)\right\} .
\end{aligned}
$$

For the gauge group $\mathrm{G}=\mathrm{SU}(2)$, we have obtained the non-Abelian Wilson loop in the path integral representation,

$$
\begin{aligned}
& W^{C}[\mathcal{A}]:=\operatorname{tr}\left[\mathcal{P} \exp \left(i \oint_{C} \mathcal{A}_{\mu}^{A}(x) T^{A} d x^{\mu}\right)\right] \\
= & \int\left[d \mu_{C}(\mathbf{n})\right] \exp \left(i J \oint_{C} d x^{\mu} \operatorname{tr}\left\{\sigma_{3}\left[U \mathcal{A}_{\mu}(x) U^{\dagger}+\frac{i}{g} U \partial_{\mu} U^{\dagger}\right]\right\}\right) \\
= & \int\left[d \mu_{C}(\mathbf{n})\right] \exp \left(i J \oint d t \operatorname{tr}\left\{\sigma_{3}\left[U \mathcal{A}(t) U^{\dagger}+\frac{i}{g} U \frac{d}{d t} U^{\dagger}\right]\right\}\right),
\end{aligned}
$$

where $J$ is the spin of the representation of the Wilson loop considered. This is a special case of the NAST of Diakonov and Petrov which will be explained in the next section.

\subsection{Non-Abelian Stokes theorem in the general case}

We give the results of Diakonov and Petrov [18, 19] in the most general form in the following. 
Definition: Let $C$ be a given curve $x_{\mu}=x_{\mu}(t)$ parameterized by $t$ where the values of the parameter $t_{1}, t_{2}$ correspond to the end points of the curve. We define the path-ordered exponent (POE) of the YM field $\mathcal{A}_{\mu}(x):=\mathcal{A}_{\mu}^{A}(x) T^{A}$ by

$$
W_{\alpha, \beta}^{C}\left(t_{2}, t_{1}\right):=\left[\mathcal{P} \exp \left(i \int_{x\left(t_{1}\right)}^{x\left(t_{2}\right)} \mathcal{A}_{\mu}^{A}(x) T^{A} d x^{\mu}\right)\right]_{\alpha, \beta} .
$$

Introducing the tangent component of the YM field along the loop,

$$
\mathcal{A}(t):=\mathcal{A}_{\mu}(x) \frac{d x^{\mu}}{d t}=\mathcal{A}_{\mu}^{A}(x) T^{A} \frac{d x^{\mu}}{d t},
$$

we can write the POE as

$$
W_{\alpha, \beta}^{C}\left(t_{2}, t_{1}\right):=\left[\mathcal{P} \exp \left(i \int_{t_{1}}^{t_{2}} \mathcal{A}(t) d t\right)\right]_{\alpha, \beta} .
$$

The POE $(3.50)$ is defined by the power-series expansion,

$$
W_{\alpha, \beta}^{C}\left(t_{2}, t_{1}\right)=\sum_{n=0}^{\infty} \int d \tau_{1} \cdots \int d \tau_{n}\left[i \mathcal{A}\left(\tau_{1}\right) \cdots i \mathcal{A}\left(\tau_{n}\right)\right]_{\alpha, \beta},
$$

where $t_{2} \geq \tau_{1} \geq \cdots \geq \tau_{n} \geq t_{1}$.

Theorem 18, 19: Consider the non-Abelian group $G$ and the maximal torus group $H$ of $G$. Define $T^{A}$ to be the generators of the representation $J: T^{A} T^{A}=J(J+1)$. Let $\mathcal{H}_{i}(i=1, \cdots, r)$ be the generators of the Cartan subalgebra of the Lie algebra $\mathcal{G}$ of $G$ and the $r$-dimensional vector $\mathbf{m}$ be the highest weight of the representation $J$ with $r$ being the rank of the gauge group $G$. Then the POE is written in the path integral form,

$$
\begin{aligned}
& W_{\alpha, \beta}^{C}\left(t_{2}, t_{1}\right) \\
= & \int d U_{1} \int d U_{2} \sum_{J^{\prime}, M^{\prime}}\left(2 J^{\prime}+1\right) D_{\alpha M^{\prime}}^{\left(J^{\prime}\right)}\left(U_{2}^{\dagger}\right) D_{M^{\prime} \beta}^{\left(J^{\prime}\right)}\left(U_{1}\right) \\
& \times \int_{U\left(t_{1}\right)=U_{1}}^{U\left(t_{2}\right)=U_{2}}[d U(t)] \exp \left(i J \int_{x\left(t_{1}\right)}^{x\left(t_{2}\right)} d x^{\mu} \operatorname{tr}\left\{m_{i} \mathcal{H}_{i}\left[U \mathcal{A}_{\mu}(x) U^{\dagger}+\frac{i}{g} U \partial_{\mu} U^{\dagger}\right]\right\}\right) \\
= & \int d U_{1} \int d U_{2} \sum_{J^{\prime}, M^{\prime}}\left(2 J^{\prime}+1\right) D_{\alpha M^{\prime}}^{\left(J^{\prime}\right)}\left(U_{2}^{\dagger}\right) D_{M^{\prime} \beta}^{\left(J^{\prime}\right)}\left(U_{1}\right) \\
& \times \int_{U\left(t_{1}\right)=U_{1}}^{U\left(t_{2}\right)=U_{2}}[d U(t)] \exp \left(i J \int_{t_{1}}^{t_{2}} d t \operatorname{tr}\left\{m_{i} \mathcal{H}_{i}\left[U \mathcal{A}(t) U^{\dagger}+\frac{i}{g} U \frac{d}{d t} U^{\dagger}\right]\right\}\right) .
\end{aligned}
$$

Here $d U$ is the invariant Haar measure on $G / H$ and $D_{M M^{\prime}}^{T}(U)$ are the Wigner $D$ function which expresses finite rotation in the representation $J$,

$$
R(U)|J, M\rangle=\sum_{M^{\prime}=-J}^{+J}\left|J, M^{\prime}\right\rangle D_{M^{\prime} M}^{(J)}(U), \quad D_{M M^{\prime}}^{(J)}(U):=\left\langle J, M|R(U)| J, M^{\prime}\right\rangle .
$$

In particular, in the spinor representation $D_{M M^{\prime}}^{1 / 2}(U)=U_{M M^{\prime}}$. 
According to the above theorem, the POE is written as a functional integral over all gauge transformations $U(t)$ of the given potential $\mathcal{A}(t)$, projected into matrix representation $\alpha, \beta$. From the above theorem, a version of NAST is given as follows. The Wilson loop, i.e. the trace of POE along a closed loop $C$ has the form,

$$
\begin{aligned}
& W^{C}[\mathcal{A}]:=\operatorname{tr}\left[\mathcal{P} \exp \left(i \oint_{C} \mathcal{A}_{\mu}^{A}(x) T^{A} d x^{\mu}\right)\right] \\
= & \int[d U(t)] \exp \left(i J \oint_{C} d x^{\mu} \operatorname{tr}\left\{m_{i} \mathcal{H}_{i}\left[U \mathcal{A}_{\mu}(x) U^{\dagger}+\frac{i}{g} U \partial_{\mu} U^{\dagger}\right]\right\}\right) \\
= & \int[d U(t)] \exp \left(i J \oint d t \operatorname{tr}\left\{m_{i} \mathcal{H}_{i}\left[U \mathcal{A}(t) U^{\dagger}+\frac{i}{g} U \frac{d}{d t} U^{\dagger}\right]\right\}\right) .
\end{aligned}
$$

For $G=S U(2)$, this reduces to (3.49). The formula is manifestly gauge invariant, as is the Wilson loop itself.

\section{Abelian and monopole dominance}

Now we show that the abelian and monopole dominance is deduced from the NAST in the formulation [2] of YM theory as a deformation of the MAG TFT. Making use of the NAST, we will clarify the meaning of Abelian dominance and monopole dominance in low-energy physics in QCD.

The full non-Abelian Wilson loop is defined as the path-ordered exponent. In the version of the NAST derived in the previous section, the path ordering has been removed from the expression. Instead, we must average over the Haar measure on $G / H$. The removal of path ordering is very welcome, since it is rather difficult to treat the path ordering. As a result, there appears the field tensor introduced by 'tHooft and Polyakov in connection with magnetic monopoles. This indicates an intimate connection between the magnetic monopole and quark confinement. In what follows, explicit calculations are performed only for $G=S U(2)$. However, the generalization to $G=S U(N)$ is straightforward.

\subsection{Magnetic monopole in YM theory}

The non-perturbative study of YM theory in the MAG goes as follows, see [1] for more details. First of all, gauge field configurations are constructed by performing the local gauge transformation (2.7) such that the gauge rotated field $\mathcal{A}_{\mu}^{U}(x)$ minimize the functional $\mathcal{R}\left[\mathcal{A}^{U}\right]$ where

$$
\mathcal{R}[\mathcal{A}]:=\int d^{D} x \operatorname{tr}_{\mathcal{G} \backslash \mathcal{H}}\left[\frac{1}{2} \mathcal{A}_{\mu}(x) \mathcal{A}_{\mu}(x)\right] .
$$

Here the trace is taken over the Lie algebra $\mathcal{G} \backslash \mathcal{H}$. In the differential form, this implies that $\mathcal{A}_{\mu}^{U}(x)$ satisfies the the gauge-fixing condition (2.20). Next, the Abelian (or diagonal) field $a_{\mu}^{U}$ and its field strength $f_{\rho \sigma}$ are extracted from the non-Abelian 
gauge field according to

$$
\begin{aligned}
a_{\mu}^{U}(x) & :=\operatorname{tr}\left[T^{3} \mathcal{A}_{\mu}^{U}(x)\right], \\
f_{\mu \nu}^{U}(x) & :=\partial_{\mu} a_{\nu}^{U}(x)-\partial_{\nu} a_{\mu}^{U}(x) .
\end{aligned}
$$

The magnetic current $k_{\mu}$ is defined from the diagonal part by

$$
k_{\mu}(x)=\partial_{\nu} \tilde{f}_{\mu \nu}^{U}(x), \quad \tilde{f}_{\mu \nu}(x):=\frac{1}{2} \epsilon_{\mu \nu \rho \sigma} f_{\rho \sigma}(x) .
$$

The magnetic charge is calculated from the magnetic current as

$$
g_{m}\left(V^{(3)}\right)=\int_{V^{(3)}} d^{3} \sigma_{\mu} k_{\mu}=\int_{V^{(3)}} d^{3} \sigma_{\mu} \partial_{\nu} \tilde{f}_{\mu \nu}^{\Omega}=\int_{S^{(2)}=\partial V^{(3)}} d^{2} \sigma_{\mu \nu} \tilde{f}_{\mu \nu}^{\Omega} .
$$

In the usual Abelian gauge theory (i.e., Maxwell theory), the magnetic monopole current vanishes identically due to the Bianchi identity,

$$
\epsilon_{\mu \nu \rho \sigma} \partial^{\mu} f^{\rho \sigma}(x) \equiv 0, \quad f_{\mu \nu}(x):=\partial_{\mu} a_{\nu}(x)-\partial_{\nu} a_{\mu}(x)
$$

as long as the field variable $a_{\mu}(x)$ is non-singular. In other words, in order to obtain a non-trivial magnetic current in Abelian gauge theory, we need to introduce the singularity into the Abelian gauge theory. This fact is well known from the study of Dirac magnetic monopole. In the non-Abelian gauge theory, the singularity is produced by partially fixing the gauge $G / H$ and leaving the Abelian subgroup $H$ of the original non-Abelian gauge group $G$ unbroken. The partial gauge fixing leads to the singularity which is sufficient to generate the magnetic monopole. This is an idea of Abelian projection by 't Hooft [4. The MAG leaves the maximal torus group $H=U(1)^{N-1}$ unbroken. This is why the YM theory can have magnetic monopoles even in the absence of the Higgs scalar field. It is well known that the YM theory in Euclidean space has instanton solutions, although the pure YM theory does not have any non-trivial classical (stable) soliton solution in four-dimensional Minkowski spacetime. It is still in dispute whether the instanton configuration alone can produce sufficient string tension for the quark confinement. The relationship between the magnetic monopole and instanton has been discussed in [1, 2], see also references cited there.

Substituting (2.7) into (4.3), we have

$$
a_{\mu}^{U}(x):=\operatorname{tr}\left[T^{3} \mathcal{A}_{\mu}^{U}(x)\right]=n^{A}(x) \mathcal{A}_{\mu}^{A}(x)+a_{\mu}^{\Omega}(x),
$$

where we have used (3.45) and defined

$$
a_{\mu}^{\Omega}(x):=\Omega_{\mu}^{3}(x):=\operatorname{tr}\left[T^{3} \Omega_{\mu}(x)\right], \quad \Omega_{\mu}(x):=\frac{i}{g} U(x) \partial_{\mu} U^{\dagger}(x) .
$$

Note that (4.7) has the same form as the argument of the exponent in the NAST (3.49). Therefore the NAST (3.49) for the Wilson loop is rewritten as

$$
W^{C}[\mathcal{A}]=\int\left[d \mu_{C}(\mathbf{n})\right] \exp \left(i J \oint_{C} d x^{\mu} a_{\mu}^{U}(x)\right),
$$


and the expectation value of the the Wilson loop is given by

$$
\left\langle W^{C}[\mathcal{A}]\right\rangle_{Y M}=\int\left[d \mu_{C}(\mathbf{n})\right]\left\langle\exp \left(i J \oint_{C} d x^{\mu} a_{\mu}^{U}(x)\right)\right\rangle_{Y M} .
$$

In the previous article [2] we have calculated the expectation value

$$
\left\langle W^{C}\left[a^{\Omega}\right]\right\rangle_{Y M}=\left\langle\exp \left(i J \oint_{C} d x^{\mu} a_{\mu}^{\Omega}(x)\right)\right\rangle_{Y M}
$$

of the diagonal Wilson loop,

$$
W^{C}\left[a^{\Omega}\right]=\exp \left(i J \oint_{C} d x^{\mu} a_{\mu}^{\Omega}(x)\right), \quad a_{\mu}^{\Omega}(x):=\Omega_{\mu}^{3}(x) .
$$

Now, the difference between the abelian (diagonal) Wilson loop (4.12) and the full non-Abelian Wilson loop (4.9) has become clear. The diagonal Wilson loop is obtained from the full non-Abelian Wilson loop by neglecting the $n^{A}(x) \mathcal{A}_{\mu}^{A}(x)$ part and the functional integral $\int[d \mu(\mathbf{n})]$ along the loop $C$. Therefore, the deviation of the diagonal Wilson loop from the full Wilson loop can be determined by estimating the effect from $n^{A}(x) \mathcal{V}_{\mu}^{A}(x)$.

If the gauge field $\mathcal{A}_{\mu}(x)$ is not singular, the first piece $U(x) \mathcal{A}_{\mu}(x) U^{\dagger}(x)$ of $\mathcal{A}_{\mu}^{U}(x)$ is non-singular and does not give rise to magnetic current. On the contrary, the second piece $\Omega_{\mu}(x)$ does give the non-vanishing magnetic monopole current (see e.g. [1]). According to Monte Carlo simulation on the lattice [10], the magnetic monopole part gives the most dominant contribution in various quantities characterizing the lowenergy physics of QCD, e.g., string tension, chiral condensate, topological charge, etc. This phenomenon is called the magnetic monopole dominance.

Therefore, it is expected that the most important degrees of freedom for the lowenergy physics comes from the second piece $\Omega_{\mu}(x)$ of $\mathcal{A}_{\mu}^{U}(x)$ rather than the first piece, $U(x) \mathcal{A}_{\mu}(x) U^{\dagger}(x)$. Therefore, we have decomposed the YM theory into two parts, i.e., the contribution from the part $\Omega_{\mu}(x)$ and the remaining part in section 2 .

Standing on this viewpoint, we recall the calculation of the abelian (diagonal) field strength in four-dimensional YM theory. The identity [囬] for $\Omega_{\mu}$,

$$
\partial_{\mu} \Omega_{\nu}(x)-\partial_{\nu} \Omega_{\mu}(x)=i g\left[\Omega_{\mu}(x), \Omega_{\nu}(x)\right]+\frac{i}{g} U(x)\left[\partial_{\mu}, \partial_{\nu}\right] U^{\dagger}(x) .
$$

leads to

$$
f_{\mu \nu}^{\Omega}(x):=\partial_{\mu} \Omega_{\nu}^{3}(x)-\partial_{\nu} \Omega_{\mu}^{3}(x)=C_{\mu \nu}^{[\Omega]}(x)+\frac{i}{g}\left(U(x)\left[\partial_{\mu}, \partial_{\nu}\right] U^{\dagger}(x)\right)^{(3)},
$$

where

$$
\begin{aligned}
C_{\mu \nu}^{[\Omega]} & :=\left(i g\left[\Omega_{\mu}, \Omega_{\nu}\right]\right)^{(3)}=g \epsilon^{a b 3} \Omega_{\mu}^{a} \Omega_{\nu}^{b}=i g\left(\Omega_{\mu}^{+} \Omega_{\nu}^{-}-\Omega_{\mu}^{-} \Omega_{\nu}^{+}\right) \\
& =\frac{1}{g} \sin \theta\left(\partial_{\mu} \theta \partial_{\nu} \varphi-\partial_{\mu} \varphi \partial_{\nu} \theta\right) .
\end{aligned}
$$

Note that $C_{\mu \nu}^{[\Omega]}$ is generated from the off-diagonal gluon fields, $\Omega_{\mu}^{1}, \Omega_{\mu}^{2}$. 
We can identity the first and second parts of right-hand-side (RHS) of (4.14) with the the magnetic monopole and the Dirac string contributions respectively. This is clearly seen by writing (4.14) explicitly using Euler angles,

$$
f_{\mu \nu}^{\Omega}=-\frac{1}{g} \sin \theta\left(\partial_{\mu} \theta \partial_{\nu} \varphi-\partial_{\mu} \varphi \partial_{\nu} \theta\right)+\frac{1}{g}\left(\left[\partial_{\mu}, \partial_{\nu}\right] \chi+\cos \theta\left[\partial_{\mu}, \partial_{\nu}\right] \varphi\right) .
$$

The magnetic charge is given by

$$
g_{m}\left(V^{(3)}\right)=\frac{1}{2 g} \int_{S^{(2)}} d^{2} \sigma_{\rho \sigma} \epsilon_{\mu \nu \rho \sigma} \sin \theta\left(\partial_{\mu} \theta \partial_{\nu} \varphi-\partial_{\mu} \varphi \partial_{\nu} \theta\right)
$$

The magnetic charge (4.18) is quantized [1], since the integrand is the Jacobian from $S^{2}$ to $S^{2}$ and

$$
\Pi_{2}(S U(2) / U(1))=\Pi_{2}\left(S^{2}\right)=Z .
$$

Then the magnetic charge $g_{m}$ satisfies the Dirac quantization condition,

$$
g_{m}=\frac{2 \pi n}{g}, \quad \text { or } \quad g g_{m}=2 \pi n(n \in Z) .
$$

We can give the second definition of the magnetic charge $g_{m}$ as the contribution from the Dirac string,

$$
g_{D S}\left(V^{(3)}\right)=\frac{1}{2 g} \int_{S^{(2)}} d^{2} \sigma_{\rho \sigma} \epsilon_{\mu \nu \rho \sigma}\left(\left[\partial_{\mu}, \partial_{\nu}\right] \chi+\cos \theta\left[\partial_{\mu}, \partial_{\nu}\right] \varphi\right) .
$$

If we choose $\chi=-\varphi$ (resp. $\chi=+\varphi$ ) using residual $\mathrm{U}(1)$ gauge invariance, then the Dirac string appears on the negative (resp. positive) $Z$ axis, i.e., $\theta=\pi$ (resp. $\theta=0$ ). In this case, the surface integral (4.21) reduces to the line integral around the string,

$$
g_{D S}\left(V^{(3)}\right)=\frac{1}{2 g} \int_{S^{(2)}} d \sigma_{\mu \nu} \epsilon_{\mu \nu \rho \sigma}\left[\partial_{\rho}, \partial_{\sigma}\right] \varphi(x)=-\frac{1}{2 g} \int_{S^{(1)}} d \sigma_{\mu \nu \rho} \epsilon_{\mu \nu \rho \sigma} \partial_{\rho} \varphi(x) .
$$

This gives the same result (4.18) but with the minus sign, which is in consistent with

$$
\Pi_{1}(U(1))=Z \text {. }
$$

Actually, two descriptions (4.18) and (4.21) are equivalent and the above argument can be generalized to more general gauge group, as suggested from

$$
\Pi_{2}\left(S U(N) / U(1)^{N-1}\right)=\Pi_{1}\left(U(1)^{N-1}\right)=Z^{N-1} .
$$

Eq. (4.13) implies

$$
\mathcal{F}_{\mu \nu}^{U}(x):=\partial_{\mu} \Omega_{\nu}(x)-\partial_{\nu} \Omega_{\mu}(x)-i g\left[\Omega_{\mu}(x), \Omega_{\nu}(x)\right] \equiv \frac{i}{g} U(x)\left[\partial_{\mu}, \partial_{\nu}\right] U^{\dagger}(x),
$$

if the contribution from $U(x) \mathcal{A}_{\mu}(x) U^{\dagger}(x)$ is completely neglected. Here the RHS is identified with the contribution from the Dirac string. The existence of Dirac string in the RHS of (4.25) reflects the fact that the field strength $\mathcal{F}_{\mu \nu}^{U}(x)$ does contain the magnetic monopole contribution. Thus we have obtained a gauge theory with magnetic monopoles starting from the YM theory (without any scalar field). Therefore, MAG enables us to deduce the magnetic monopole without introducing the scalar field, in contrast to the 't Hooft-Polyakov monopole [34, 35. 


\subsection{Magnetic monopole and non-Abelian Stokes theorem}

We show that the magnetic monopole does appear in the gauge invariant Wilson loop of YM theory through the NAST. The NAST gives a gauge-invariant description of the magnetic monopole in YM theory.

The second term in the exponent (3.49) can be rewritten as a surface integral inside the closed contour of the Wilson loop. The parameterization of $\mathrm{SU}(2)$ matrix $U$ by the Euler angles leads to

$$
\Omega_{\mu}^{3}(x):=\operatorname{tr}\left(\sigma_{3} \frac{i}{g} U(x) \partial_{\mu} U^{\dagger}(x)\right)=\frac{1}{g}\left[\partial_{\mu} \chi(x)+\cos \theta(x) \partial_{\mu} \varphi(x)\right] .
$$

Then the second term in the exponent of (3.49) reads

$$
\begin{aligned}
i J \oint_{C} d x^{\mu} \Omega_{\mu}^{3}(x) & =i J \oint_{C} d x^{\mu} \operatorname{tr}\left(\sigma_{3} \frac{i}{g} U(x) \partial_{\mu} U^{\dagger}(x)\right) \\
& =i \frac{J}{g} \oint_{C} d x^{\mu}\left[\partial_{\mu} \chi(x)+\cos \theta(x) \partial_{\mu} \varphi(x)\right]
\end{aligned}
$$

This is rewritten as a surface integral using the standard Abelian Stokes theorem,

$$
i J \oint_{C} d x^{\mu} \Omega_{\mu}^{3}(x)=i J \int_{S} d^{2} z \epsilon_{\mu \nu}\left(\partial_{\mu} \Omega_{\nu}^{3}-\partial_{\nu} \Omega_{\mu}^{3}\right)=i J \int_{S} d^{2} z \epsilon_{\mu \nu} f_{\mu \nu}^{\Omega} .
$$

By making use of a unit vector $\mathbf{n}$, this is further rewritten as [2]

$$
\begin{aligned}
i J \oint_{C} d x^{\mu} \Omega_{\mu}^{3}(x) & =i \frac{J}{g} \int_{S} d^{2} z \epsilon^{A B C} \epsilon_{\mu \nu} n^{A} \partial_{\mu} n^{B} \partial_{\nu} n^{C} \\
& =i \frac{J}{g} \int_{S} d^{2} z \epsilon_{\mu \nu} \mathbf{n} \cdot\left(\partial_{\mu} \mathbf{n} \times \partial_{\nu} \mathbf{n}\right) \\
& =i \frac{8 \pi J}{g} Q_{S},
\end{aligned}
$$

where $Q$ is the topological charge of the $\mathbf{n}$ field [2] in the area $S$,

$$
Q_{S}:=\frac{1}{8 \pi} \int_{S} d^{2} z \epsilon_{\mu \nu} \mathbf{n} \cdot\left(\partial_{\mu} \mathbf{n} \times \partial_{\nu} \mathbf{n}\right)=\frac{1}{4 \pi} \int_{S} d^{2} \sigma_{\mu \nu} \mathbf{n} \cdot\left(\partial_{\mu} \mathbf{n} \times \partial_{\nu} \mathbf{n}\right) .
$$

On the other hand, the first term in the exponent (3.49) is rewritten as

$$
i J \oint d x^{\mu} \operatorname{tr}\left\{\sigma_{3}\left[U \mathcal{V}_{\mu}(x) U^{\dagger}\right]\right\}=i J \oint d x^{\mu} \mathcal{V}_{\mu}^{A}(x) n^{A}(x)=i J \oint d t \mathcal{V}^{A}(t) n^{A}(t)
$$

Thus we obtain another version of NAST,

$$
\begin{aligned}
& W^{C}[\mathcal{A}] \\
= & \int[d \mu(\mathbf{n})] \exp \left\{i J\left[\oint_{C} d x^{\mu} n^{A}(x) \mathcal{V}_{\mu}^{A}(x)+\frac{1}{g} \int_{S} d^{2} \sigma_{\mu \nu} \mathbf{n} \cdot\left(\partial_{\mu} \mathbf{n} \times \partial_{\nu} \mathbf{n}\right)\right]\right\} \\
= & \int[d \mu(\mathbf{n})] \exp \left\{i J\left[\oint_{C} d t n^{A}(t) \mathcal{V}^{A}(t)+\frac{1}{g} \int_{S} d^{2} \sigma_{\mu \nu} \mathbf{n} \cdot\left(\partial_{\mu} \mathbf{n} \times \partial_{\nu} \mathbf{n}\right)\right]\right\} .
\end{aligned}
$$


Furthermore, the first term in the exponent is rewritten as

$$
i J \oint_{C} d x^{\mu} n^{A}(x) \mathcal{V}_{\mu}^{A}(x)=i J \int_{S} d^{2} \sigma^{\mu \nu} \frac{1}{2}\left[\partial_{\mu}\left(n^{A}(x) \mathcal{V}_{\nu}^{A}(x)\right)-\partial_{\nu}\left(n^{A}(x) \mathcal{V}_{\mu}^{A}(x)\right)\right]
$$

Therefore, a manifestly gauge-invariant formula of the non-Abelian Wilson loop has been obtained [18, 19]

$$
W^{C}[\mathcal{A}]=\int[d \mu(\mathbf{n})] \exp \left\{i \frac{J}{2} \int_{S} d^{2} \sigma^{\mu \nu} G_{\mu \nu}(x)\right\}
$$

with the gauge-invariant tensor field [36],

$$
G_{\mu \nu}(x):=\partial_{\mu}\left(n^{A}(x) \mathcal{V}_{\nu}^{A}(x)\right)-\partial_{\nu}\left(n^{A}(x) \mathcal{V}_{\mu}^{A}(x)\right)-\frac{1}{g} \mathbf{n}(x) \cdot\left(\partial_{\mu} \mathbf{n}(x) \times \partial_{\nu} \mathbf{n}(x)\right)
$$

This is nothing but the 'tHooft tensor [34, 35, 36] if we identify $n^{A}$ with the direction of the elementary Higgs field,

$$
\hat{\phi}^{A}:=\phi^{A} /|\phi|, \quad|\phi|:=\sqrt{\phi^{A} \phi^{A}}
$$

The tensor (4.35) gives a SU(2) gauge-invariant definition for the electromagnetic field tensor, since using the covariant derivative,

$$
D_{\mu}^{A B}:=\partial_{\mu} \delta^{A B}-g \epsilon^{A B C} \mathcal{A}_{\mu}^{C}
$$

it is rewritten as

$$
\begin{aligned}
G_{\mu \nu}(x) & :=n^{A}(x) \mathcal{F}_{\mu \nu}^{A}(x)-\frac{1}{g} \epsilon^{A B C} \mathbf{n}^{A}(x)\left(D_{\mu} \mathbf{n}(x)\right)^{B}\left(D_{\nu} \mathbf{n}(x)\right)^{C} \\
& =\operatorname{tr}\left[\mathbf{n}(x) \mathcal{F}_{\mu \nu}(x)-\frac{1}{g} \mathbf{n}(x)\left(D_{\mu} \mathbf{n}(x)\right)\left(D_{\nu} \mathbf{n}(x)\right)\right]
\end{aligned}
$$

where we have used

$$
\left[\sigma^{A}, \sigma^{B}\right]=2 i \epsilon^{A B C} \sigma^{C}, \quad \operatorname{tr}\left(\sigma^{A} \sigma^{B}\right)=2 \delta^{A B}, \quad \operatorname{tr}\left(\sigma^{A} \sigma^{B} \sigma^{C}\right)=2 i \epsilon^{A B C} .
$$

Note that both terms in (4.39) are gauge invariant, because under the gauge transformation $\mathbf{n}(x), D_{\mu} \mathbf{n}(x)$ and $\mathcal{F}_{\mu \nu}(x)$ transform as the adjoint representation,

$$
\begin{aligned}
\mathbf{n}(x) & \rightarrow U(x) \mathbf{n}(x) U^{\dagger}(x), \\
D_{\mu} \mathbf{n}(x) & \rightarrow U(x) D_{\mu} \mathbf{n}(x) U^{\dagger}(x), \\
\mathcal{F}_{\mu \nu}(x) & \rightarrow U(x) \mathcal{F}_{\mu \nu}(x) U^{\dagger}(x) .
\end{aligned}
$$

The Wilson loop is the evolution operator for spin $J \mathbf{n}$ in a time-dependent "external (magnetic) field" $\mathcal{V}_{\mu}(t)$ and the Wess-Zumino term,

$$
S_{W Z}:=\int d^{2} \sigma_{\mu \nu} \mathbf{n} \cdot\left(\partial_{\mu} \mathbf{n} \times \partial_{\nu} \mathbf{n}\right)
$$


fixes the representation to which the spin belongs. The non-Abelian Wilson loop measures the flux of magnetic monopole through the area $S$ enclosed by the Wilson loop $C$ where the magnetic monopole is generated from the topological non-trivial configuration of $\mathbf{n}(x)$.

Unlike the usual electromagnetic field tensor, the tensor (4.35) has a dual with non-zero divergence, i.e., non-vanishing magnetic monopole current,

$$
k_{\mu}=\frac{1}{2} \epsilon_{\mu \nu \rho \sigma} \partial^{\nu} G^{\rho \sigma}=\frac{1}{2 g} \epsilon_{\mu \nu \rho \sigma} \partial^{\nu} \mathbf{n} \cdot\left(\partial_{\rho} \mathbf{n} \times \partial_{\sigma} \mathbf{n}\right)=\frac{1}{2 g} \epsilon_{\mu \nu \rho \sigma} \partial^{\nu}\left[\mathbf{n} \cdot\left(\partial_{\rho} \mathbf{n} \times \partial_{\sigma} \mathbf{n}\right)\right] .
$$

The monopole current $k_{\mu}$ is a conserved topological current, $\partial^{\mu} k_{\mu} \equiv 0$. Although the $k_{\mu}$ is written as a total divergence, it can give non-vanishing magnetic charge (4.18),

$$
g_{m}=\int_{V^{(3)}} d^{3} x k_{0}=\frac{2 \pi n}{g} .
$$

In the region where $\mathbf{n}=(0,0,1)$, the 't Hooft tensor reads

$$
G_{\mu \nu}(x)=\partial_{\mu} \mathcal{V}_{\nu}^{3}(x)-\partial_{\nu} \mathcal{V}_{\mu}^{3}(x)
$$

and the magnetic current vanishes identically, $k_{\mu} \equiv 0$.

\subsection{Abelian magnetic monopole dominance}

Note that we can replace $\mathcal{A}_{\mu}$ (appearing in the argument of the exponent in the NAST (3.49) ) with $\mathcal{V}_{\mu}$ which has been defined in the reformulation of the YM theory. This is shown as follows. If $\mathcal{A}_{\mu}(x)$ is the gauge rotation of $\mathcal{V}_{\mu}(x)$ by $\tilde{U}(x)$,

$$
\mathcal{A}_{\mu}(x):=\tilde{U}(x) \mathcal{V}_{\mu}(x) \tilde{U}^{\dagger}(x)+\frac{i}{g} \tilde{U}(x) \partial_{\mu} \tilde{U}^{\dagger}(x)=\mathcal{V}_{\mu}^{\tilde{U}}(x)
$$

then

$$
\begin{aligned}
\mathcal{A}_{\mu}^{U}(x) & :=U(x) \mathcal{A}_{\mu}(x) U^{\dagger}(x)+\frac{i}{g} U(x) \partial_{\mu} U^{\dagger}(x) \\
& =(U(x) \tilde{U}(x)) \mathcal{V}_{\mu}(x)(U(x) \tilde{U}(x))^{\dagger}+\frac{i}{g}(U(x) \tilde{U}(x)) \partial_{\mu}(U(x) \tilde{U}(x))^{\dagger} \\
& =\mathcal{V}_{\mu}^{U \tilde{U}}(x) .
\end{aligned}
$$

As the new matrix $U \tilde{U}$ is also an element of $G$, we can absorb this change into the invariant Haar measure $\left[d \mu_{C}(\mathbf{n})\right]$. Therefore we can write the NAST (4.48) as

$$
\begin{aligned}
& W^{C}[\mathcal{A}]:=\operatorname{tr}\left[\mathcal{P} \exp \left(i \oint_{C} \mathcal{A}_{\mu}^{A}(x) T^{A} d x^{\mu}\right)\right] \\
= & \int\left[d \mu_{C}(\mathbf{n})\right] \exp \left(i J \oint_{C} d x^{\mu} \operatorname{tr}\left\{\sigma_{3}\left[U \mathcal{V}_{\mu}(x) U^{\dagger}+\frac{i}{g} U \partial_{\mu} U^{\dagger}\right]\right\}\right) \\
= & \int\left[d \mu_{C}(\mathbf{n})\right] \exp \left(i J \oint d t \operatorname{tr}\left\{\sigma_{3}\left[U \mathcal{V}(t) U^{\dagger}+\frac{i}{g} U \frac{d}{d t} U^{\dagger}\right]\right\}\right),
\end{aligned}
$$


and the expectation value of the Wilson loop reads

$$
\begin{aligned}
& \left\langle W^{C}[\mathcal{A}]\right\rangle_{Y M} \\
= & \int d \mu_{C}(\mathbf{n})\left\langle\operatorname { e x p } i J [ \oint _ { C } d x ^ { \mu } n ^ { A } ( x ) \mathcal { V } _ { \mu } ^ { A } ( x ) + \frac { 1 } { g } \int _ { S } d ^ { 2 } z \epsilon _ { \mu \nu } \mathbf { n } \cdot ( \partial _ { \mu } \mathbf { n } \times \partial _ { \nu } \mathbf { n } ) ] \left(\phi_{\mu}\right.\right.
\end{aligned}
$$

where the expectation value is written according to (2.36) as

$$
\begin{aligned}
& \left\langle\exp \left\{i J\left[\oint_{C} d x^{\mu} n^{A}(x) \mathcal{V}_{\mu}^{A}(x)+\frac{1}{g} \int_{S} d^{2} z \epsilon_{\mu \nu} \mathbf{n} \cdot\left(\partial_{\mu} \mathbf{n} \times \partial_{\nu} \mathbf{n}\right)\right]\right\}\right\rangle_{Y M} \\
& =Z_{Y M}^{-1} \int[d U][d \mathcal{C}][d \overline{\mathcal{C}}][d \phi] e^{i S_{T F T}\left[\Omega_{\mu}, \mathcal{C}, \overline{\mathcal{C}}, \phi\right]} e^{i \frac{J}{g} \int_{S} d^{2} z \epsilon_{\mu \nu} \mathbf{n} \cdot\left(\partial_{\mu} \mathbf{n} \times \partial_{\nu} \mathbf{n}\right)} \\
& \times \int\left[d \mathcal{V}_{\mu}\right][d \gamma][d \bar{\gamma}][d \beta] e^{i S_{p Y M}[\mathcal{V}, \gamma, \bar{\gamma}, \beta]} \\
& \times e^{i \int d^{D} x\left(i \mathcal{V}_{\mu}^{A} \mathcal{M}_{\mu}^{A}[U]+\frac{i}{2} \mathcal{V}_{\mu}^{A} \mathcal{V}_{\mu}^{B} \mathcal{K}^{A B}[U]\right)} e^{i J \oint_{C} d x^{\mu} n^{A}(x) \mathcal{V}_{\mu}^{A}(x)}
\end{aligned}
$$

The denominator, i.e. the partition function $Z_{Y M}$ is equal to

$$
Z_{Y M}=\left\langle\left\langle e^{i \int d^{D} x\left(i \mathcal{V}_{\mu}^{A} \mathcal{M}_{\mu}^{A}[U]+\frac{i}{2} \mathcal{V}_{\mu}^{A} \mathcal{V}_{\mu}^{B} \mathcal{K}^{A B}[U]\right)}\right\rangle_{p Y M} Z_{p Y M}\right\rangle_{T F T} Z_{T F T}
$$

where $Z_{p Y M}$ is the partition function of perturbative sector of the YM theory,

$$
Z_{p Y M}:=\int\left[d \mathcal{V}_{\mu}\right][d \gamma][d \bar{\gamma}][d \beta] e^{i S_{p Y M}[\mathcal{V}, \gamma, \bar{\gamma}, \beta]}
$$

and $Z_{T F T}$ is the partition function of the TFT,

$$
Z_{T F T}:=\int[d U][d \mathcal{C}][d \overline{\mathcal{C}}][d \phi] e^{i S_{T F T}\left[\Omega_{\mu}, \mathcal{C}, \overline{\mathcal{C}}, \phi\right]}
$$

The numerator is equal to

$$
\begin{aligned}
& \left\langle\left\langle e^{i \int d^{D} x\left(i \mathcal{V}_{\mu}^{A} \mathcal{M}_{\mu}^{A}[U]+\frac{i}{2} \mathcal{V}_{\mu}^{A} \mathcal{V}_{\mu}^{B} \mathcal{K}^{A B}[U]\right)} e^{i J \oint_{C} d x^{\mu} n^{A}(x) \mathcal{V}_{\mu}^{A}(x)}\right\rangle_{p Y M} Z_{p Y M}\right. \\
& \left.\quad \times e^{i \frac{J}{g} \int_{S} d^{2} z \epsilon_{\mu \nu} \mathbf{n} \cdot\left(\partial_{\mu} \mathbf{n} \times \partial_{\nu} \mathbf{n}\right)}\right\rangle_{T F T} Z_{T F T} .
\end{aligned}
$$

The expectation value of the Wilson loop is given by the ratio, (4.54)/(4.51). In (4.51), the argument of the exponential including $\mathcal{M}_{\mu}^{A}[U]$ and $\mathcal{K}^{A B}[U]$ (2.32) is written in the BRST exact form,

$$
e^{i \int d^{D} x\left(i \mathcal{V}_{\mu}^{A} \mathcal{M}_{\mu}^{A}[U]+\frac{i}{2} \mathcal{V}_{\mu}^{A} \mathcal{V}_{\mu}^{B} \mathcal{K}^{A B}[U]\right)}=e^{i\left\{Q_{B}, *\right\}} .
$$

Expanding this exponential and using the fact that

$$
Q_{B}^{\dagger}=Q_{B}, \quad Q_{B}|0\rangle_{T F T}=0, \quad Q_{B}^{2}=0
$$


we see that the partition function in the absence of external sources has the decomposition,

$$
Z_{Y M}=Z_{p Y M} Z_{T F T}
$$

Thus the expectation value of the Wilson loop is written as

$$
\begin{gathered}
\left\langle W^{C}[\mathcal{A}]\right\rangle_{Y M}=\left\langle\left\langle e^{i \int d^{D} x\left(i \mathcal{V}_{\mu}^{A} \mathcal{M}_{\mu}^{A}[U]+\frac{i}{2} \mathcal{V}_{\mu}^{A} \mathcal{V}_{\mu}^{B} \mathcal{K}^{A B}[U]\right)} e^{i J \oint_{C} d x^{\mu} n^{A}(x) \mathcal{V}_{\mu}^{A}(x)}\right\rangle_{p Y M}\right. \\
\left.\times e^{i \frac{J}{g} \int_{S} d^{2} z \epsilon_{\mu \nu} \mathbf{n} \cdot\left(\partial_{\mu} \mathbf{n} \times \partial_{\nu} \mathbf{n}\right)}\right\rangle_{T F T}
\end{gathered}
$$

By repeating similar arguments, the Wilson loop is cast into the form,

$$
\left\langle W^{C}[\mathcal{A}]\right\rangle_{Y M}=\left\langle\left\langle e^{i J \oint_{C} d x^{\mu} n^{A}(x) \mathcal{V}_{\mu}^{A}(x)}\right\rangle_{p Y M} e^{i \frac{J}{g} \int_{S} d^{2} z \epsilon_{\mu \nu} \mathbf{n} \cdot\left(\partial_{\mu} \mathbf{n} \times \partial_{\nu} \mathbf{n}\right)}\right\rangle_{T F T} .
$$

The perturbative part is expanded into

$$
\begin{aligned}
& \left\langle e^{i J \oint_{C} d x^{\mu} n^{A}(x) \mathcal{V}_{\mu}^{A}(x)}\right\rangle_{p Y M} \\
& =1-\frac{1}{2} J^{2} \oint_{C} d x^{\mu} \oint_{C} d y^{\nu} n^{A}(x) n^{B}(y)\left\langle\mathcal{V}_{\mu}^{A}(x) \mathcal{V}_{\nu}^{B}(y)\right\rangle_{p Y M}+O\left(g^{4}\right),
\end{aligned}
$$

where we have used $\left\langle\mathcal{V}_{\mu}^{A}(x)\right\rangle_{p Y M}=0$. Then we can write

$$
\begin{aligned}
\left\langle W^{C}[\mathcal{A}]\right\rangle= & \left\langle e^{i \frac{J}{g} \int_{S} d^{2} z \epsilon_{\mu \nu} \mathbf{n} \cdot\left(\partial_{\mu} \mathbf{n} \times \partial_{\nu} \mathbf{n}\right)}\right\rangle_{T F T} \\
& \times\left[1-\frac{1}{2} J^{2} \oint_{C} d x^{\mu} \oint_{C} d y^{\nu}\left\langle\mathcal{V}_{\mu}^{A}(x) \mathcal{V}_{\nu}^{B}(y)\right\rangle_{p Y M}\right. \\
& \left.\times \frac{\left\langle n^{A}(x) n^{B}(y) e^{i \frac{J}{g} \int_{S} d^{2} z \epsilon_{\mu \nu} \mathbf{n} \cdot\left(\partial_{\mu} \mathbf{n} \times \partial_{\nu} \mathbf{n}\right)}\right\rangle_{T F T}}{\left\langle e^{i \frac{J}{g} \int_{S} d^{2} z \epsilon_{\mu \nu} \mathbf{n} \cdot\left(\partial_{\mu} \mathbf{n} \times \partial_{\nu} \mathbf{n}\right)}\right\rangle_{T F T}}+O\left(g^{4}\right)\right] \cdot(
\end{aligned}
$$

Owing to the dimensional reduction, the expectation value of the diagonal Wilson loop $\left\langle e^{i \frac{J}{g} \int_{S} d^{2} z \epsilon_{\mu \nu} \mathbf{n} \cdot\left(\partial_{\mu} \mathbf{n} \times \partial_{\nu} \mathbf{n}\right)}\right\rangle_{T F T}$ in the RHS of (4.61) in the four-dimensional TFT (2.39) is reduced to that in the two-dimensional NLSM (2.40), when $C$ is planar,

$$
\left\langle W^{C}\left[a^{\Omega}\right]\right\rangle_{T F T_{4}}=\left\langle e^{i \frac{J}{g} \int_{S} d^{2} z \epsilon_{\mu \nu} \mathbf{n} \cdot\left(\partial_{\mu} \mathbf{n} \times \partial_{\nu} \mathbf{n}\right)}\right\rangle_{T F T_{4}}=\left\langle e^{i \frac{J}{g} \int_{S} d^{2} z \epsilon_{\mu \nu} \mathbf{n} \cdot\left(\partial_{\mu} \mathbf{n} \times \partial_{\nu} \mathbf{n}\right)}\right\rangle_{N L S M_{2}}
$$

The quantity $Q_{S}[\mathbf{n}]$ defined by

$$
Q_{S}[\mathbf{n}]:=\frac{1}{8 \pi} \int_{S} d^{2} z \epsilon_{\mu \nu} \mathbf{n} \cdot\left(\partial_{\mu} \mathbf{n} \times \partial_{\nu} \mathbf{n}\right)=n_{+}^{i n}-n_{-}^{i n},
$$

is an integer and counts the instanton-anti-instanton charge $\left(n_{+}^{i n}-n_{-}^{i n}\right)$ inside the Wilson loop. By summing up the instanton and anti-instanton contributions in the 
two-dimensional NLSM, we have obtained the area law for the diagonal Wilson loop in the previous article [2],

$$
\left\langle W^{C}\left[a^{\Omega}\right]\right\rangle_{T F T_{4}}=\left\langle e^{i \frac{J}{g} 8 \pi Q_{S}[\mathbf{n}]}\right\rangle_{N L S M_{2}} \cong e^{-\sigma_{A b e l} A(C)},
$$

where $A(C)$ is the area enclosed by the Wilson loop $C$. We call the coefficient in the area decay $\sigma_{\text {Abel }}$ the Abelian string tension. The naive instanton calculus based on the dilute instanton gas approximation [2] leads to

$$
\sigma_{\text {Abel }}=2 B e^{-S_{1}}\left[1-\cos \left(\frac{2 \pi q}{g}\right)\right], \quad S_{1}=\frac{4 \pi^{2}}{g^{2}},
$$

where $B$ is a constant with the mass-squared dimension, $B \sim m_{A}^{2}$ and $S_{1}=4 \pi^{2} / g^{2}$ is the action for one instanton. Here we have neglected to write the perimeter decay part which can be generated by instantons and anti-instantons located just on the perimeter of the Wilson loop.

Now we proceed to estimate the remaining terms. To simplify the perturbation calculation in the RHS of (4.61), we take the Feynman gauge in the perturbative sector where the propagator reads

$$
\begin{aligned}
\left\langle\mathcal{V}_{\mu}^{A}(x) \mathcal{V}_{\nu}^{B}(y)\right\rangle_{p Y M} & =\delta^{A B} \delta_{\mu \nu} G(x, y) \\
G(x, y) & =\int \frac{d^{4} p}{(2 \pi)^{4}} e^{i p(x-y)} \frac{g^{2}}{p^{2}}=\frac{g^{2}}{4 \pi^{2}} \frac{1}{|x-y|^{2}}
\end{aligned}
$$

Then we obtain

$$
\begin{array}{r}
\left\langle W^{C}[\mathcal{A}]\right\rangle_{Y M}=\left\langle e^{i \frac{J}{g} 8 \pi Q_{S}[\mathbf{n}]}\right\rangle_{N L S M_{2}}\left[1-\frac{1}{2} J^{2} \oint_{C} d x^{\mu} \oint_{C} d y^{\mu} G(x, y)\right. \\
\left.\times \frac{\left\langle\mathbf{n}(x) \cdot \mathbf{n}(y) e^{i \frac{J}{g} 8 \pi Q_{S}[\mathbf{n}]}\right\rangle_{N L S M_{2}}}{\left\langle e^{i \frac{J}{g} 8 \pi Q_{S}[\mathbf{n}]}\right\rangle_{N L S M_{2}}}+O\left(g^{4}\right)\right] .
\end{array}
$$

where we have used the dimensional reduction [2] for the correlation function,

$$
\begin{aligned}
& \left\langle\mathbf{n}(x) \cdot \mathbf{n}(y) e^{i \frac{J}{g} 8 \pi Q_{S}[\mathbf{n}]}\right\rangle_{T F T_{4}}=\left\langle\mathbf{n}(x) \cdot \mathbf{n}(y) e^{i \frac{J}{g} 8 \pi Q_{S}[\mathbf{n}]}\right\rangle_{N L S M_{2}}, \\
& x, y \in C=\partial S \subset R^{2}
\end{aligned}
$$

for the planar Wilson loop $C$,

A naive estimate for the expectation value in $\mathrm{NLSM}_{2}$ is given by considering the instanton contribution. The instanton solution in NLSM is given by the field configuration $\mathbf{n}$ such that $\mathbf{n}$ approaches the same value $\mathbf{n}^{(0)}$ at infinity (see [2])

$$
\mathbf{n}(x) \rightarrow \mathbf{n}^{(0)} \quad(|x| \rightarrow \infty)
$$

where $\mathbf{n}^{(0)}$ is any unit vector, $\mathbf{n}^{(0)} \cdot \mathbf{n}^{(0)}=1$. Therefore, for large Wilson loop $C$

$$
\mathbf{n}(x) \cdot \mathbf{n}(y) \rightarrow \mathbf{n}^{(0)} \cdot \mathbf{n}^{(0)}=1, \quad(x, y \in C=\partial S) .
$$


Here the configuration $\mathbf{n}^{(0)} \equiv(0,0,1)$ corresponds to the topological trivial case, $Q=0$. The precise estimate of (4.69) can be done using the large $N$ expansion for $\mathrm{O}(\mathrm{N})$ NLSM. In fact, for not so large $|x-y|$, the two-point correlation function behaves as (see e.g. [37)

$$
\langle\mathbf{n}(x) \cdot \mathbf{n}(y)\rangle_{N L S M_{2}}=\left[1-\frac{N-2}{2 \pi} \frac{1}{\beta} \ln \frac{|x-y|}{\epsilon}\right]^{\frac{N-1}{N-2}},
$$

where $\epsilon$ is a short distance cutoff.

It turns out that the contribution of the last term in (4.68) gives the perimeter law correction to the area law. For large $T \gg R \gg 1$, we have (see Appendix and 38, 39, 40, 41, 42, 43,)

$$
-\frac{1}{2} \oint_{C} d x^{\mu} \oint_{C} d y^{\mu} G(x, y) \cong-\frac{g^{2}}{2 \pi^{2}} \frac{T+R}{\epsilon}+\frac{g^{2}}{4 \pi} \frac{T}{R}+\frac{g^{2}}{2 \pi^{2}} \ln \frac{R}{\epsilon} .
$$

It should be remarked that the perimeter decay in (4.73) comes from the contribution of the coincident point, $x=y$, (after regularization, $|x-y| \cong \epsilon \ll 1$, see Appendix). Similarly we can evaluate the higher-order terms which give the running coupling constant in consistent with the asymptotic freedom. These contributions from the perturbative sector should be compared with the conventional calculation based on the perturbative QCD [38].

Exponentiating the contributions from the power-series expansion [38], we obtain for $g$ small,

$$
\begin{aligned}
\left\langle W^{C}[\mathcal{A}]\right\rangle_{Y M} & \cong\left\langle e^{i \frac{J}{g} 8 \pi Q}\right\rangle_{N L S M_{2}} e^{-C g^{2}(R+T)+\frac{g^{2}}{4 \pi} \frac{T}{R}+C^{\prime}} \\
& =e^{-\sigma_{A b e l} R T-C g^{2}(R+T)+\frac{g^{2}}{4 \pi} \frac{T}{R}+C^{\prime}}
\end{aligned}
$$

where $C$ and $C^{\prime}$ are constants. The full non-Abelian string tension is defined by

$$
\sigma:=-\lim _{A(C) \rightarrow \infty} \frac{1}{A(C)} \ln \left\langle W^{C}[\mathcal{A}]\right\rangle_{Y M}
$$

where $A(C)$ is the area enclosed by the Wilson loop. For a rectangular loop with side lengths $R$ and $T, A(C)=R T$. The above result shows that the Abelian (diagonal) Wilson loop obeys the same area law as the Non-Abelian Wilson loop and that the area law of the full non-Abelian Wilson loop is deduced from the magnetic monopole contribution for the diagonal Wilson loop, $\left\langle e^{i \frac{J}{g} 8 \pi Q}\right\rangle_{T F T}$. This implies the monopole dominance in the string tension of QCD under the MAG. The deviation of the Abelian string tension from the full non-Abelian string tension is given by

$$
\begin{aligned}
\sigma-\sigma_{\text {Abel }} & =-\lim _{A(C) \rightarrow \infty} \frac{1}{A(C)} \ln \left\langle W^{C}[\mathcal{A}]\right\rangle_{Y M}+\lim _{A(C) \rightarrow \infty} \frac{1}{A(C)} \ln \left\langle e^{i \frac{J}{g} 8 \pi Q}\right\rangle_{T F T} \\
& =\lim _{R, T \rightarrow \infty} \frac{C g^{2}(R+T)-\frac{g^{2}}{4 \pi} \frac{T}{R}-C^{\prime}}{R T}=0 .
\end{aligned}
$$


Hence the deviation of the string tension comes from the finite size effect of the Wilson loop. For sufficiently large Wilson loop, $\sigma>\sigma_{\text {Abel }}$ and the off-diagonal contribution to the string tension vanishes as $R, T \rightarrow \infty$. In the large Wilson loop limit $R, T \rightarrow \infty$, the Abelian string tension coincides exactly with the full non-Abelian string tension, $\sigma=\sigma_{\text {Abel }}$. Thus Abelian and monopole dominance for the string tension can be proved under the MAG according to the formulation of the YM theory as a deformation of the TFT. It is rather straightforward to extend the above strategy to the case, $G=S U(N), N \geq 3$.

It should be remarked that, if the massive decay of the correlation function (4.72) for large separation $|x-y| \gg 1(x, y \in C)$ is incorporated in the above evaluation, the Coulomb part $\frac{g^{2}}{4 \pi} \frac{T}{R}$ in (4.73) will be replaced by the Yukawa part, $\frac{g^{2}}{4 \pi} \frac{T}{R} e^{-m R}$, where $m$ is the mass of the $\mathbf{n}$ field of the NLSM. However, this effect does not change the conclusion for the string tension. In fact, the perimeter part is generated from the coincidence limit $|x-y| \ll 1$. Furthermore, if we averaged $\mathbf{n}(x) \cdot \mathbf{n}(y)$ over all possible configurations, we would have obtained,

$$
\int d \mu_{C}(\mathbf{n}) \mathbf{n}(x) \cdot \mathbf{n}(y)=\delta^{(2)}(x-y),
$$

from a fact that $\mathbf{n}(x)$ and $\mathbf{n}(y)$ are independent for the measure $d \mu_{C}(\mathbf{n})$. Consequently, only the coincident contribution survives in (4.68), which leads to the perimeter decay correction alone in (4.76) (without the Coulomb or Yukawa part).

Monte Carlo simulation of lattice gauge theory supports the finite size effect as a deviation of the string tension, as argued by Suganuma et al. [44] using the computerassisted analytical study.

\subsection{Abelian dominance}

According to the NAST, one must average over all gauge transformation in the coset $\mathrm{SU}(2) / \mathrm{U}(1)$. Abelian dominance is the statement that in the true quantum vacuum, the contributions to the $n$ average is approximated by the Abelian projection. This replaces $n^{A}(x) \mathcal{V}_{\mu}^{A}(x)$ with $n^{3}(x) \mathcal{V}_{\mu}^{3}(x)$. In our standpoint, the contribution to the area law and non-vanishing string tension comes from the topological term, i.e., the second term in the exponent (4.32), because the first term can only give the perturbative correction around non-trivial topological sector. Actually, the first term may give a long-range Coulomb potential in the topological trivial sector $Q=0$. Therefore, according to the reformulation of YM theory as a deformation of MAG TFT, the dominance of topological non-trivial term (the second term) is an immediate consequence of the formulation. This implies the monopole dominance in the string tension. In addition, the Abelian dominance is an immediate consequence of the APEGT together with the above considerations.

Here it should be remarked that the Abelian (diagonal) Wilson loop in the nonAbelian gauge theory is not the same as the Wilson loop in the Abelian gauge theory. In the Abelian U(1) gauge theory, the Wilson loop is given by [3]

$$
W^{C}[a]:=\exp \left(i q \oint_{C} a_{\mu}(x) d x^{\mu}\right)
$$




$$
=\exp \left(i q \oint_{C} d x^{\mu}\left[v_{\mu}(x)+\frac{i}{g} U \partial_{\mu} U^{\dagger}\right]\right), \quad U(x)=e^{i \varphi(x)} \in U(1)
$$

\subsection{Gluon self-interactions in the perturbative sector}

In the new reformulation of gauge theory [2], QCD has been identified with a perturbative deformation of the TQFT. In this reformulation the non-perturbative dynamics of QCD is saturated by the TQFT. This identification will be meaningful at least in the low energy physics (including the quark confinement) by the following reasons. In principle, of course, additional non-perturbative dynamics could possibly come from the self-interaction among the gluon fields reflecting the non-Abelian nature of the gauge group. However, additional non-perturbative contributions to quark confinement are expected to be rather small, if any. This is because the recent numerical simulations [9, 10] of lattice gauge theory with the maximal Abelian gauge fixing have confirmed the magnetic monopole dominance as well as the Abelian dominance in low-energy physics of QCD for various quantities including the string tension.

Another reason from the theoretical viewpoint is as follows. As shown in [1], we can integrate out the off-diagonal gluon fields in QCD to obtain the low-energy effective gauge theory of QCD, i.e. APEGT. Note that the APEGT is the Abelian gauge theory. Hence, at this stage, we do not worry so seriously about the remaining gluon self-interactions which are identified as the perturbative deformation to the TQFT in the reformulation of QCD. Then the non-vanishing magnetic monopole current $k_{\mu}(x)$ is generated from the diagonal Abelian part $a_{\mu}^{\Omega}(x)$ according to [1]. In addition, the result of the previous article [2] shows that the condensation of magnetic monopoles in the four-dimensional QCD is deduced from the instanton (or vortex) condensation in the two-dimensional NLSM obtained from the dimensional reduction of the four-dimensional TQFT. Therefore, the low-energy dynamics of QCD in the MAG is considered to be described well by the TQFT or its dimensional reduction, i.e., NLSM. In the low-energy region where the APEGT is meaningful, therefore, quark confinement will follow from these considerations without much difficulties by combining the results of the previous articles [1, 2] with the result of this article.

\section{A Evaluation of Wilson integral}

In order to evaluate the Wilson integral, we choose a rectangular contour with side lengths $R$ and $T$. Then we have

$$
\begin{aligned}
& \oint_{C} d x_{\mu} \oint_{C} d y_{\mu} \frac{1}{|x-y|^{2}} \\
& =-2 \int_{0}^{T} d t^{\prime} \int_{0}^{T} d t^{\prime \prime} \frac{1}{R^{2}+\left(t^{\prime}-t^{\prime \prime}\right)^{2}}-2 \int_{0}^{R} d r^{\prime} \int_{0}^{R} d r^{\prime \prime} \frac{1}{T^{2}+\left(r^{\prime}-r^{\prime \prime}\right)^{2}} \\
& \quad+2 \int_{0}^{T} d t^{\prime} \int_{0}^{T} d t^{\prime \prime} \frac{1}{\left(t^{\prime}-t^{\prime \prime}\right)^{2}}+2 \int_{0}^{R} d r^{\prime} \int_{0}^{R} d r^{\prime \prime} \frac{1}{\left(r^{\prime}-r^{\prime \prime}\right)^{2}} .
\end{aligned}
$$

Note that $d x_{\mu} d y_{\mu}$ implies that only integrations between parallel sides give a contribution, i.e., no contribution between neighboring sides where $d x_{\mu} d y_{\mu}=0$. In the line 
integrals in the first (resp. second) lines of (A.1), $x$ and $y$ run over opposite (resp. same) sides of the rectangle. The integral over the opposite side is

$$
\int_{0}^{T} d t^{\prime} \int_{0}^{T} d t^{\prime \prime} \frac{1}{R^{2}+\left(t^{\prime}-t^{\prime \prime}\right)^{2}}=\frac{2 T}{R} \arctan \frac{T}{R}-\ln \left(1+\frac{T^{2}}{R^{2}}\right) .
$$

On the other hand, the integrals over the same sides diverge. So we omit the integral around the singularity by introducing the infinitesimal parameter $\epsilon$,

$$
\int_{0}^{T} d t^{\prime} \int_{0}^{T} d t^{\prime \prime} \frac{1}{\left(t^{\prime}-t^{\prime \prime}\right)^{2}}=2 \int_{0}^{T-\epsilon} d t^{\prime} \int_{t^{\prime}+\epsilon}^{T} d t^{\prime \prime} \frac{1}{\left(t^{\prime}-t^{\prime \prime}\right)^{2}}=2 \frac{T-\epsilon}{\epsilon}+2 \ln \frac{\epsilon}{T} .
$$

Summing all terms yields

$$
\begin{aligned}
& \oint_{C} d x_{\mu} \oint_{C} d y_{\mu} \frac{1}{|x-y|^{2}} \\
& =-2\left[\frac{2 T}{R} \arctan \frac{T}{R}+\frac{2 R}{T} \arctan \frac{R}{T}-\ln \left(1+\frac{T^{2}}{R^{2}}\right)-\ln \left(1+\frac{R^{2}}{T^{2}}\right)\right. \\
& \left.\quad-2 \frac{T+R-2 \epsilon}{\epsilon}-2 \ln \frac{\epsilon}{T}-2 \ln \frac{\epsilon}{R}\right] .
\end{aligned}
$$

For simplicity, we consider the rectangle which is much larger in temporal direction than the spatial direction, $T \gg R(\gg \epsilon)$,

$$
\oint_{C} d x_{\mu} \oint_{C} d y_{\mu} \frac{1}{|x-y|^{2}} \cong-2\left[\pi \frac{T}{R}+2 \ln \frac{R}{\epsilon}-2 \frac{T+R-2 \epsilon}{\epsilon}\right] .
$$

Then we obtain

$$
-\frac{1}{2} \oint_{C} d x_{\mu} \oint_{C} d y_{\mu} \frac{g^{2}}{4 \pi^{2}} \frac{1}{|x-y|^{2}} \cong-\frac{g^{2}}{2 \pi^{2}} \frac{T+R}{\epsilon}+\frac{g^{2}}{4 \pi} \frac{T}{R}+\frac{g^{2}}{2 \pi^{2}} \ln \frac{R}{\epsilon} .
$$

In RHS of (A.6) the first term exhibits the perimeter law. The second term corresponds to the Coulomb law. The constant term represents the self-energy of a regularized point charge. This should be subtracted during the course of renormalization. On the lattice, $\epsilon$ is replaced with the lattice spacing. The last logarithmic divergent term does not occur for the path $C$ with continuous tangent [43].

In the Abelian gauge theory, the static potential is given by

$$
\begin{aligned}
V(R) & :=-\lim _{T \rightarrow \infty} \frac{1}{T} \ln \left\langle W^{C}[v]\right\rangle \\
& =-\lim _{T \rightarrow \infty} \frac{1}{T} \ln \left\langle\exp \left[i \oint_{C} d x^{\mu} v_{\mu}(x)\right]\right\rangle_{p U(1)} \\
& =-\lim _{T \rightarrow \infty} \frac{1}{T} \ln \exp \left[-\frac{1}{2} \oint_{C} d x^{\mu} \oint_{C} d y^{\nu}\left\langle v_{\mu}(x) v_{\nu}(y)\right\rangle_{p U 1)}\right] \\
& =-\lim _{T \rightarrow \infty} \frac{1}{T} \ln \exp \left(-\frac{1}{2} \oint_{C} d x_{\mu} \oint_{C} d y_{\mu} \frac{g^{2}}{4 \pi^{2}} \frac{1}{|x-y|^{2}}\right) \\
& \cong \frac{g^{2}}{2 \pi^{2}} \frac{1}{\epsilon}-\frac{g^{2}}{4 \pi} \frac{1}{R},
\end{aligned}
$$


where the last term gives the Coulomb potential. The above evaluation of the Wilson loop shows that the perimeter law follows from the contribution, $x=y$. For more details, see 38, 39, 40, 41, 42, 43.

\section{Acknowledgments}

I would like to thank Volodya Miransky for suggestions on revising the article and Giovanni Prosperi for pointing out the misleading expressions in the first version of this article. I am also grateful to Mauro Zeni for sending many comments on the articles [2, 3] and Dmitri Antonov for informing me of the information on more nonAbelian Stokes theorems. This work is supported in part by the Grant-in-Aid for

Scientific Research from the Ministry of Education, Science and Culture.

\section{References}

[1] K.-I. Kondo, Abelian-projected effective gauge theory of QCD with asymptotic freedom and quark confinement, Chiba Univ. Preprint, CHIBA-EP-99, hepth/9709109 (revised), Phys. Rev. D 57, 7467-7487 (1998).

K.-I. Kondo, Talk given at YKIS'97: Non-perturbative QCD, 2-12 December 1997, Kyoto, Chiba Univ. Preprint, CHIBA-EP-104, hep-th/9803063, Prog. Theor. Phys. Supplement, No. 130, in press.

[2] K.-I. Kondo, Yang-Mills Theory as a Deformation of Topological Field Theory, Dimensional Reduction and Quark Confinement, Chiba Univ. Preprint, CHIBA-EP-103, hep-th/9801024 (revised), Phys. Rev. D, in press.

[3] K.-I. Kondo, Existence of confinement phase in quantum electrodynamics, Chiba Univ. Preprint, CHIBA-EP-105, hep-th/9803133, Phys. Rev. D, in press.

[4] G. 't Hooft, Topology of the gauge condition and new confinement phases in non-Abelian gauge theories, Nucl. Phys. B 190 [FS3], 455-478 (1981).

[5] G.S. Bali, C. Schlichter and K. Schilling, Probing the QCD Vacuum with Static Sources in Maximal Abelian Projection, hep-lat/9802005.

[6] P.A.M. Dirac, Quantized singularities in the electromagnetic field, Proc. Roy. Soc., London, A 133, 60-72 (1931).

P.A.M. Dirac, The theory of magnetic poles, Phys. Rev. 74, 817-830 (1948).

[7] T.T. Wu and C.N. Yang, Concept of nonintegrable phase factors and global formulation of gauge fields, Phys. Rev. D 12, 3845-3857 (1975).

T.T. Wu and C.N. Yang, Dirac monopole without strings: monopole harmonics, Nucl. Phys. B 107, 365 (1976).

[8] Z.F. Ezawa and A. Iwazaki, Abelian dominance and quark confinement in YangMills theories, Phys. Rev. D 25, 2681-2689 (1982).

Abelian dominance and quark confinement in Yang-Mills theories, II. Oblique confinement and $\eta^{\prime}$ mass, Phys. Rev. D 26, 631-647 (1982). 
[9] T. Suzuki and I. Yotsuyanagi, Possible evidence of abelian dominance in quark confinement, Phys. Rev. D 42, 4257-4260 (1990).

S. Hioki, S. Kitahara, S. Kiura, Y. Matsubara, O. Miyamura, S. Ohno and T. Suzuki, Abelian dominance in SU(2) color confinement, Phys. Lett. B 272, 326332 (1991).

Monopole distribution in momentum space in $\mathrm{SU}(2)$ lattice gauge theory, Phys. Lett. B 285, 343-346 (1992).

[10] T. Suzuki, Monopole condensation in lattice SU(2) QCD, hep-lat/9506016.

R.W. Haymaker, Dual Abrikosov vortices in U(1) and SU(2) lattice gauge theories, hep-lat/9510035.

A. DiGiacomo, Monopole condensation and color confinement, hep-lat/9802008; Mechanisms for color confinement, hep-th/9603029.

M.I. Polikarpov, Recent results on the abelian projection of lattice gluodynamics, hep-lat/9609020. M.N. Chernodub and M.I. Polikarpov, Abelian projections and monopoles, hep-th/9710205.

[11] R.C. Brower, K.N. Orginos and Chung-I Tan, Magnetic monopole loop for the Yang-Mills instanton, hep-th/9610101, Phys. Rev. D 55, 6313-6326 (1997).

[12] G.S. Bali, V. Bornyakov, M. Müller-Preussker and K. Schilling, Dual Superconductor Scenario of Confinement: A Systematic Study of Gribov Copy Effects, hep-lat/9603012, Phys. Rev. D 54, 2863-2875 (1996).

[13] J. Stack, S. Neiman and R. Wensley, String Tension from Monopoles in SU(2) Lattice Gauge Theory hep-lat/9404014, Phys. Rev. D50, 3399-3405 (1994).

[14] M.B. Halpern, Field-strength and dual variable formulations of gauge theory, Phys. Rev. D 19, 517-530 (1979).

[15] N.E. Bralic, Exact computation of loop averages in two-dimensional Yang-Mills theory, Phys. Rev. D 22, 3090-3103 (1980).

[16] I.Ya. Aref'eva, Non-Abelian Stokes formula, Theor. Math. Phys. 43, 111-116 (1980). [translation, 353-356]

[17] Yu.A. Simonov, Cluster expansion, nonAbelian Stokes theorem and magnetic monopoles, Yad. Fiz. 50, 213-224 (1989) [Sov. J. Nucl. Phys. 50, 134 (1989)].

[18] D.I. Diakonov and V.Yu. Petrov, A formula for the Wilson loop, Phys. Lett. B 224, 131-135 (1989).

[19] D. Diakonov and V. Petrov, Non-Abelian Stokes theorem and quark-monopole interaction, hep-th/9606104.

[20] F.A. Lunev, Pure bosonic worldline path integral representation for fermionic determinants, non-Abelian Stokes theorem, and quasiclassical approximation in QCD, hep-th/9609166, Nucl. Phys. B494, 433-470 (1997).

[21] M. Hirayama and S. Matsubara, Stokes theorem for loop variables of nonAbelian gauge field, hep-th/9712120, Prog. Theor. Phys., to appear.

[22] J. Klauder, Path integrals and stationary-phase approximations, Phys. Rev. D 19, 2349-2356 (1979). 
[23] L.S. Schulman, Techniques and applications of path integration (John Wiley \& Sons, 1981).

[24] T. Kashiwa, Y. Ohnuki and M. Suzuki, Path integral methods (Oxford Univ. Press, 1997).

[25] E. Fradkin, Field theories of condensed matter systems (Addison-Wesley, 1991). A. Auerbach, Interacting electrons and quantum magnetism (Springer, 1994).

[26] M.V. Berry, Quantum phase factors accompanying adiabatic changes, Proc. R. Soc. Lond. A 392, 45-57 (1984).

M.V. Berry, Anticipations of the geometric phase, Physics Today, 43, No. 12 , 34-40 (1990).

[27] B. Simon, Holonomy, the quantum adiabatic theorem and Berry's phase, Phys. Rev. Lett. 51, 2167-2170 (1983).

[28] F. Wilczek and A. Zee, Appearance of gauge structure in simple dynamical systems, Phys. Rev. Lett. 24, 2111-2114 (1984).

[29] M. Stone, Born-Oppenheimer approximation and the origin of Wess-Zumino terms: some quantum-mechanical examples, Phys. Rev. D 33, 1191-1194 (1986).

[30] E. Fradkin and M. Stone, Topological terms in one- and two-dimensional quantum Heisenberg antiferromagnets, Phys. Rev. B 38, 7215-7218 (1988).

[31] Y. Aharonov and J. Anandan, Phase change during a cyclic quantum evolution, Phys. Rev. Lett. 58, 1593-1596 (1987).

J. Anandan and Y. Aharonov, Geometry of quantum evolution, Phys. Rev. Lett. 65, 1697-1700 (1990).

J. Anandan, Geometric phase for cyclic motions and the quantum state space metric, Phys. Lett. A 147, 3-8 (1990).

[32] A. Shapere and F. Wilczek, Geometric phases in physics, Advanced series in mathematical physics, Vol. 5 (World Scientific, 1989).

[33] K.-I. Kondo, A proof of quark confinement in QCD, Talk given at the 3rd International conference on Quark Confinement and the Hadron Spectrum, 712 June 1998, Jefferson Lab., Newport News, VA, USA.

[34] G. 't Hooft, Magnetic monopoles in unified gauge theories, Nucl. Phys. B 79, 276-284 (1974).

[35] A.M. Polyakov, Particle spectrum in the quantum field theory, Pisma Zh. Eksp. Teor. Fiz. 20, 430-433 (1974) [JETP Lett. 20, 194-195 (1974)].

[36] J. Arafune, P.G.O. Freund and C.J. Goebel, Topology of Higgs fields, J. Math. Phys. 16, 433-437 (1975).

[37] A.M. Polyakov, Gauge fields and strings (Harwood Academic Publishers, London, 1987).

[38] J.B. Kogut, The lattice gauge theory approach to quantum chromodynamics, Rev. Mod. Phys. 55, 775-836 (1983). 
[39] P. Becher, M. Böhm and H. Joos, Gauge theories of strong and electroweak interactions (John Wiley \& Sons, 1984).

[40] W. Fischler, Quark-antiquark potential in QCD, Nucl. Phys. B 129, 157-174 (1977).

[41] R.J. Hughes, Some comments on asymptotic freedom, Phys. Lett. B 97, 246-248 (1980).

[42] A.M. Polyakov, Gauge fields as rings of glue, Nucl. Phys. B 164, 171-188 (1979).

[43] V.S. Dotsenko and S.N. Vergeles, Renormalizability of phase factors in nonAbelian gauge theory, Nucl. Phys. B 169, 527-546 (1980).

[44] H. Suganuma, H. Ichie, A. Tanaka and K. Amemiya, Instantons and monopoles in the nonperturbative QCD, hep-lat/9804027.

H. Ichie and H. Suganuma, Semi-analytical proof of Abelian dominance on confinement in the maximally Abelian gauge, hep-lat/9807025. 